\title{
LACUNARY FRACTIONAL BROWNIAN MOTION
}

\author{
Marianne Clausel ${ }^{1}$
}

\begin{abstract}
In this paper, a new class of Gaussian field is introduced called Lacunary Fractional Brownian Motion. Surprisingly we show that usually their tangent fields are not unique at every point. We also investigate the smoothness of the sample paths of Lacunary Fractional Brownian Motion using wavelet analysis.
\end{abstract}

Mathematics Subject Classification. 42C40,26B35.

Received October 28, 2009. Revised February 22, 2010.

\section{INTRODUCTION}

In [15] and [16], Falconer generalized the concept of tangent field previously defined in [6] by Benassi et al. to study the local structure of Multifractional Brownian Motion. A field $Y$ is a weak tangent field of the random field $X$ at $x_{0}$, if there exists two decreasing sequences of non negative real numbers converging to zero $\rho_{n, 1}$ and $\rho_{n, 2}$ such that,

$$
\left\{\frac{X\left(x_{0}+\rho_{n, 1} t\right)-X\left(x_{0}\right)}{\rho_{n, 2}}\right\} \rightarrow\{Y(t)\}
$$

where convergence is defined in an appropriate sense.

In many specific cases, such as Fractional Brownian Motion (FBM) or Multifractional Brownian Motion, the tangent field is essentially unique. More precisely, there exists a field $Y$ such that the collection of all tangent fields of $X$ at $x_{0}$, called the tangent space of $X$ at $x_{0}$, equals $\langle Y\rangle$, the family of non negative scalar multiple of $Y$. In that specific case, $Y$ is called the tangent field of $X$ at $x_{0}$.

Furthermore, under the assumption of the uniqueness of the tangent field, Falconer proves that tangent fields must be self-similar at the origin and must have stationary increments almost everywhere. In the special case of Gaussian fields for which tangent field is unique at every point, it implies that almost everywhere either the tangent field is 'smooth' or it is locally 'fractal' like a FBM.

Natural questions appear: can we define a Gaussian field for which there is no uniqueness of the tangent field at any point? What is the structure of the tangent space of such a field at every point? The Lacunary Fractional Brownian Motion (LBFM) provides a simple answer to these questions. At every point, LFBM admits several tangent fields. In addition, although LBFM is a Gaussian field, we can give two explicit examples of tangent fields unlike a FBM at every point. This last property results from asymptotic self-similarity properties

\footnotetext{
Keywords and phrases. Lacunary Gaussian fields, non uniqueness of the tangent field, uniform irregularity, wavelets.

1 Laboratoire d'Analyse et de Mathématiques Appliquées, UMR 8050 du CNRS, Université Paris Est, 61 Avenue du Général de Gaulle, 94010 Créteil Cedex, France. marianne.clausel@free.fr
} 
thoroughly described in the following pages. Additionally, the sample paths of LFBM satisfy some specific regularity properties that we study using wavelets techniques.

Our plan will be as follows: in Section 2, we first briefly recall some definitions concerning FBM and Multiscale FBM. Thereafter, we introduce two new classes of Gaussian fields: Infinite Scale Fractional Brownian Motion (ISFBM in short) and a special case of ISFBM: Lacunary Fractional Brownian Motion which satisfies paradoxical self-similarity properties. In Section 4 we review some well-known facts about tangent fields. Then we prove that LFBM is a Gaussian field admitting several tangent fields at every point. In this case, our main theorem describes the structure of tangent space at every point and the related asymptotic self-similarity properties according to the different scales. In Section 5 we investigate the sample paths properties of ISFBM. More precisely, we will see that different situations can appear: the sample paths regularity associated with a sequence of scales can be all the same for every sequence or on the opposite, can depend on the chosen sequence. In the example of LFBM, this field presents different regularity properties of the sample paths according to the different sequences of scales. Section 6 is devoted to the proofs of the results stated in Sections 4 and 5 .

\section{Some Multi-scale Gaussian Models}

The aim of this Section (see Sect. 2.1) is firstly to recall basic facts about an already known multi-scale Gaussian model: the Multi-scale Fractional Brownian Motion. This field was recently introduced in [2] by Bardet and Bertrand and generalizes in some sense FBM. In Section 2.2, we introduce two new classes of multiscale Gaussian fields derived from Multiscale Fractional Brownian Motion: Infinite Scale Fractional Brownian Motion and Lacunary Fractional Brownian Motion whose definition is the main purpose of this paper.

\subsection{The Multi-scale Fractional Brownian Motion}

The Multi-scale Fractional Brownian Motion is one of the numerous generalizations of Fractional Brownian Motion. Let us recall that Fractional Brownian Motion, introduced by Kolmogorov in [20] and studied by Mandelbrot and Van Ness in [25], is the continuous mean-zero Gaussian process $\left\{B_{\underline{H}}(t)\right\}_{t \in \mathbb{R}}$ depending on a parameter $\underline{H} \in(0,1)$, called the Hurst index, with covariance kernel

$$
\mathbb{E}\left(B_{\underline{H}}(t) B_{\underline{H}}(s)\right)=|s|^{2} \underline{H}+|t|^{2} \underline{H}-|s-t|^{2} \underline{H} .
$$

This process has important applications in modelling (see [7]), for example in hydrology or in finance. Its main properties are all related to its Hurst index $\underline{H}$. More precisely, since FBM is self-similar, the sample paths regularity (related to the high frequency behavior) and the long memory properties of the increments (related to the low frequency behavior) are all driven by the same Hurst index $\underline{H}$.

In some applications, one needs more flexibility and two Hurst indices are required in order to describe the low and the high frequency behavior of the process. Let us quote an example in finance given by Bardet and Bertrand in [2]. If we are interested in the price process, statistical studies show that this process satisfies long memory properties (see [26]). Thus, at low frequencies, the Hurst index should be larger than 1/2. But financial theory assumes it is not possible to make profit without any risk. This implies that the price process is a semi-martingale and that the Hurst index should be $\underline{H}=1 / 2$ at high frequencies. So in that case one must introduce two different Hurst indices to describe the behavior of the process at high and low frequencies. Others examples can also be found in turbulence or in biomechanics (see [17] or [10]).

For this reason, new models of Gaussian fields with a Hurst index varying as a piecewise function of frequencies have been introduced implicitely by Collins et al. [10] or explicitely by Benassi and Deguy (see [5]). Both Collins et al. and Benassi and Deguy defined a process with two Hurst indices: one related to high frequencies, another related to low frequencies.

To study precisely the probabilistic properties of this class of processes, in [2] Bardet and Bertrand introduced a much more general model called Multi-scale Fractional Brownian Motion where the Hurst index is a piecewise 
function of frequency. They pick a finite number of frequencies,

$$
\omega_{0}=0<\ldots<\omega_{J+1}=+\infty
$$

a finite number of Hurst indices $H_{0}, \ldots, H_{J+1}$, and a finite number of amplitudes $\sigma_{0}, \ldots, \sigma_{J+1}$. The Multi-scale Fractional Brownian motion $\left\{B_{\omega, \mathbf{H}, \sigma}(t)\right\}_{t \in \mathbb{R}^{d}}$ is then defined by its harmonisable representation

$$
B_{\omega, \mathbf{H}, \sigma}(t)=\sum_{\ell=0}^{J} \sigma_{\ell} \int_{\omega_{\ell}<|\xi|<\omega_{\ell+1}} \frac{\mathrm{e}^{i t . \xi}-1}{|\xi|^{H_{\ell}+\frac{d}{2}}} \mathrm{~d} \widehat{W}(\xi) .
$$

Then, they study the main properties of Multi-scale Fractional Brownian Motion: regularity of the sample paths and long memory properties.

Let us be more precise about the distinction between the model proposed by Benassi and Deguy in [5] and this proposed by Bardet and Bertrand in [2]. Following the literature on change point detection (see for e.g. [11] or [4]), the model proposed by Benassi and Deguy is At Most One Change (AMOC), whether this of Bardet and Bertrand admits a spectral density with More Than One Change. It is well known in the statistical community working on abrupt change detection that there is a gap between AMOC and More Than One Change problems.

The main goal of this paper is to generalize the Multi-scale model of Bardet and Bertrand in order to recover specific properties as non uniqueness of the tangent field. To this end we introduce a new model called Infinite Scale Fractional Brownian Motion (ISFBM). Thereafter, we focus on a special case of ISFBM: Lacunary Fractional Brownian Motion (LFBM) which satisfy the required property of non-uniqueness of tangent fields at any point.

\subsection{Infinite Scale Fractional Brownian Motion and Lacunary Fractional Brownian Motion}

Our first model, Infinite Scale Fractional Brownian Motion, is a refinement of Multi-scale Fractional Brownian Motion. Our aim is to define a field presenting different behaviors according to different family of scales. For this reason, we need to consider not a finite number of band of frequencies but an infinite one. This leads us to introduce ISFBM:

Definition 2.1. Let $\mathbf{H}=\left(H_{j}\right)_{j}$ be a sequence of reals numbers satisfying simultaneously the two following conditions:

$$
\left\{\begin{array}{l}
0<\liminf _{j \rightarrow+\infty} H_{j}, \\
\limsup _{j \rightarrow+\infty} H_{j}<1,
\end{array}\right.
$$

and $\sigma=\left(\sigma_{j}\right)_{j}$ be a bounded sequence of positive real numbers. The Infinity Scale Fractional Brownian Motion $\left\{B_{\mathbf{H}, \sigma}(t)\right\}_{t \in \mathbb{R}^{d}}$ with Hurst indices $\mathbf{H}=\left(H_{j}\right)_{j}$ and amplitudes $\sigma=\left(\sigma_{j}\right)_{j}$ is the real valued Gaussian field with stationary increments whose harmonizable representation is given by

$$
B_{\mathbf{H}, \sigma}(t)=\sum_{\ell=0}^{+\infty} \sigma_{\ell} \int_{D_{\ell}} \frac{\mathrm{e}^{i t \xi}-1}{|\xi|^{H_{\ell}+\frac{d}{2}}} \mathrm{~d} \widehat{W}(\xi),
$$

where $D_{0}=B_{d}(0,1)=\{\xi,|\xi|<1\}$ and for all $\ell \geq 1, D_{\ell}=\left\{\xi, 2^{\ell-1}<|\xi|<2^{\ell}\right\}$.

Remark 2.2. One can wonder if more general model than ISFBM defined above can be considered. A natural extension could be Gaussian fields of the form

$$
B_{H, \sigma}(\xi) \int \sigma(|\xi|) \frac{\mathrm{e}^{i t \xi}-1}{|\xi|^{H(|\xi|)+\frac{d}{2}}} \mathrm{~d} \widehat{W}(\xi)
$$




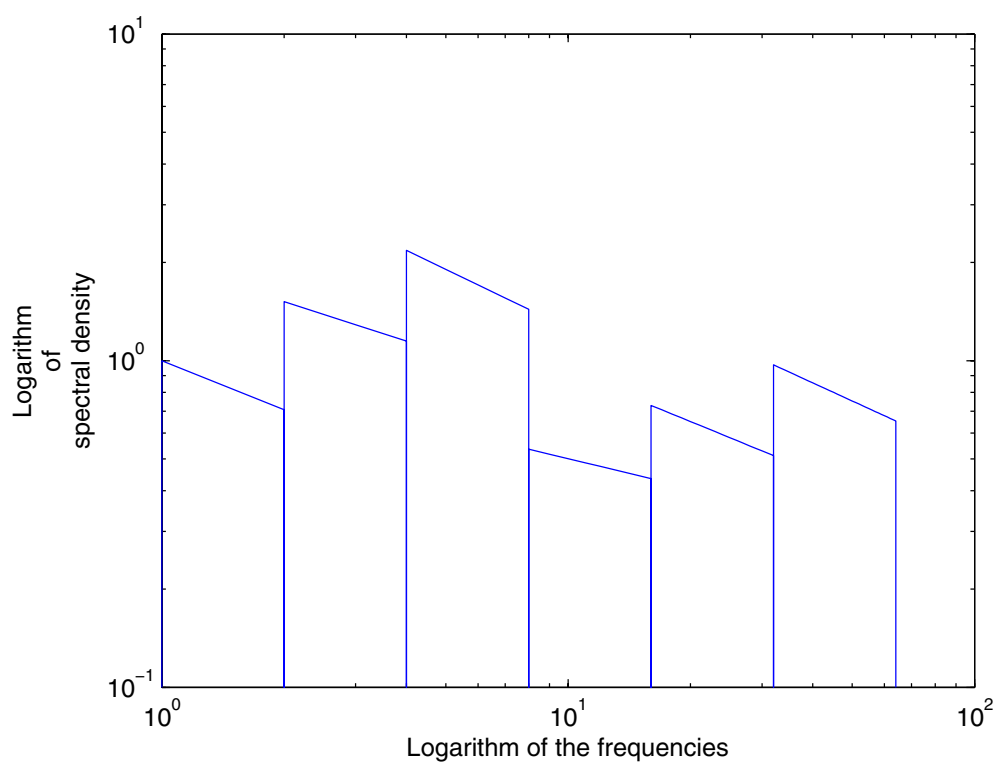

FigurE 1. An example of spectral density of ISFBM as function of the frequencies.

where $\sigma$ is a bounded function on $R_{+}$and $H$ a bounded function on $R_{+}$with values in $(0,1)$. Nevertheless, all our regularity results on ISFBM (see Sect. 5) are based on an estimate of the correlation of wavelet coefficients (see Lem. 6.3) and we encounter technical difficulties to extend this Lemma. Much work has to be done to extend our results to the more general case of fields of the form (2.3). It will be the subject of a forthcoming paper.

Remark 2.3. Remark that the two conditions

$$
\left\{\begin{array}{l}
H_{0}>0 \\
\limsup _{j \rightarrow+\infty} H_{j}<1
\end{array}\right.
$$

are necessary to prove the existence of the ISFBM with Hurst indices $\mathbf{H}=\left(H_{j}\right)_{j}$ and amplitudes $\sigma=\left(\sigma_{j}\right)_{j}$. Moreover, the additional assumption

$$
\liminf _{j \rightarrow+\infty} H_{j}<1
$$

ensures the almost sure uniform Hölderian regularity of the sample paths of the ISFBM defined by equation (2.2) (see Sect. 5).

Figure 1 just below represents an example of the spectral density of an ISFBM. Let us point out that despite our figure ISFBM is not a finite band Gaussian field.

In some specific cases, the ISFBM coincides with already known Gaussian fields. We give some examples below:

Example 2.4. The Infinity Scale Fractional Brownian Motion $\left\{B_{\mathbf{H}, \sigma}(t)\right\}_{t \in \mathbb{R}^{d}}$ with Hurst indices $\mathbf{H} \equiv \underline{H}$ and amplitudes $\sigma \equiv 1$ is the classical Fractional Brownian Motion.

Example 2.5. If there exists an integer $J$ such that, for all $j \geq J, \sigma_{j}=\sigma_{J}$ and $H_{j}=H_{J}$ then the Infinity Scale Fractional Brownian Motion $\left\{B_{\mathbf{H}, \sigma}(t)\right\}_{t \in \mathbb{R}^{d}}$ with Hurst indices $\mathbf{H}=\left(H_{j}\right)_{j}$ and amplitudes $\sigma=\left(\sigma_{j}\right)_{j}$ is a Multi-scale Fractional Brownian Motion as defined in [2].

Example 2.6. If for all integer $j, \sigma_{j}=1$, ISFBM is a special case of the Generalized Multifractional Brownian Motion introduced by Ayache and Lévy-Véhel in [1]. Let us recall that, if $\lambda>1$ is an arbitrary fixed real and 
$\mathbf{h}=\left(h_{\ell}(\cdot)\right)_{\ell}$ is a sequence of Hölder functions with values in $[a, b] \subset(0,1)$, then the GMBM with parameters $\lambda$ and $\mathbf{H}$ is the continuous Gaussian process $Y_{\mathbf{H}, \lambda}$ defined as

$$
Y_{\mathbf{H}, \lambda}(t)=\int_{0 \leq|\xi|<1} \frac{\mathrm{e}^{i t \xi}-1}{|\xi|^{h_{\ell}(t)+\frac{d}{2}}} \mathrm{~d} \widehat{W}(\xi)+\sum_{\ell=0}^{+\infty} \int_{\lambda^{\ell} \leq|\xi|<\lambda^{\ell+1}} \frac{\mathrm{e}^{i t \xi}-1}{|\xi|^{h_{\ell}(t)+\frac{d}{2}}} \mathrm{~d} \widehat{W}(\xi) .
$$

If $\lambda=2$ and for all $\ell, h_{\ell}(\cdot) \equiv H_{\ell}$, we recover the Infinity Scale Fractional Brownian Motion $\left\{B_{\mathbf{H}, \sigma}(t)\right\}_{t \in \mathbb{R}^{d}}$ with Hurst indices $\mathbf{H}=\left(H_{j}\right)_{j}$ and amplitudes $\sigma \equiv 1$.

In the following, we will not focus on the case $\sigma \equiv 1$. On the opposite, we will be especially interested in another special case: the LFBM. In that case, we allow some values of the amplitude sequence $\sigma=\left(\sigma_{j}\right)_{j}$ to vanish. Thus we recover some very special properties, as non unicity of tangent fields, reflecting that the behavior of LFBM is different according to the different families of scales. Our aim is to introduce a Gaussian field admitting different behaviors according two different families of scales related to two different Hurst indices. We are given a first Hurst index $\underline{H} \in(0,1)$. Our approach consists in introducing a first family of frequencies (denoted $2^{\ell_{n}}$ in the following) and a first Gaussian field

$$
X_{1}(x)=\sum_{n=0}^{+\infty} \int_{D_{\ell_{n}}} \frac{\mathrm{e}^{i x \xi}-1}{|\xi|^{\underline{H}+\frac{d}{2}}} \mathrm{~d} \widehat{W}_{\xi} .
$$

At frequencies $2^{\ell_{n}}$, the behavior of this field is clearly driven by $\underline{H}$. If the sequence $2^{\ell_{n}}$ is sufficiently lacunary, we will prove that there exists a sequence of intermediary frequencies $2^{j_{n}}$ - depending both on the lacunarity degree $\tau$ of the sequence $2^{\ell_{n}}$ and on $\underline{H}$ - where the influence of frequencies located at the scale $2^{\ell_{n}}$ is minimal. We thus can add to the previous Gaussian field another Gaussian part:

$$
X_{2}(x)=\sum_{n=0}^{+\infty} \int_{D_{j_{n}}} \frac{\mathrm{e}^{i x \xi}-1}{|\xi|^{\bar{H}+\frac{d}{2}}} \mathrm{~d} \widehat{W}_{\xi}
$$

The Hurst index $\bar{H}$ is well-chosen to ensure that $X_{2}$ is always negligible with respect to $X_{1}$ except at scales $2^{-j_{n}}$ and is related to $\underline{H}, \tau$. We then define a new model - Lacunary Fractional Brownian Motion - which satisfies all the required properties. Let us be more precise:

Definition 2.7. Let $\tau>1$ and $\underline{H} \in(0,1)$. Define $\bar{H}$ as

$$
\bar{H}=\frac{\tau \underline{H}}{1-\underline{H}+\tau \underline{H}} .
$$

Define the two following sequences $\left(j_{n}\right)_{n \in \mathbb{N}}$ et $\left(\ell_{n}\right)_{n \in \mathbb{N}}$ by $j_{0}=1$ and for all integer $n \geq 1$ as

$$
\ell_{n}=\frac{\bar{H}}{\underline{H}} j_{n}+\tau \log \left(j_{n}\right), j_{n+1}=\frac{1-\underline{\underline{H}}}{1-\bar{H}} \ell_{n}+\tau \log \left(\ell_{n}\right) .
$$

The Lacunary Fractional Brownian Motion with lower Hurst index $\underline{H}$ and lacunary index $\tau$ is the real valued Gaussian field with stationary increments whose harmonizable representation is given by:

$$
B_{\underline{H}, \tau}(x)=\sum_{n=0}^{+\infty} \int_{D_{j_{n}}} \frac{\mathrm{e}^{i x \xi}-1}{|\xi|^{\bar{H}+\frac{d}{2}}} \mathrm{~d} \widehat{W}_{\xi}+\sum_{n=0}^{+\infty} \int_{D_{\ell_{n}}} \frac{\mathrm{e}^{i x \xi}-1}{|\xi| \underline{H}+\frac{d}{2}} \mathrm{~d} \widehat{W}_{\xi} .
$$

Figure 2 just below represents an example of the spectral density of an LFBM with Hurst index $H=0.5$ and lacunarity index $\tau=1.6$. 


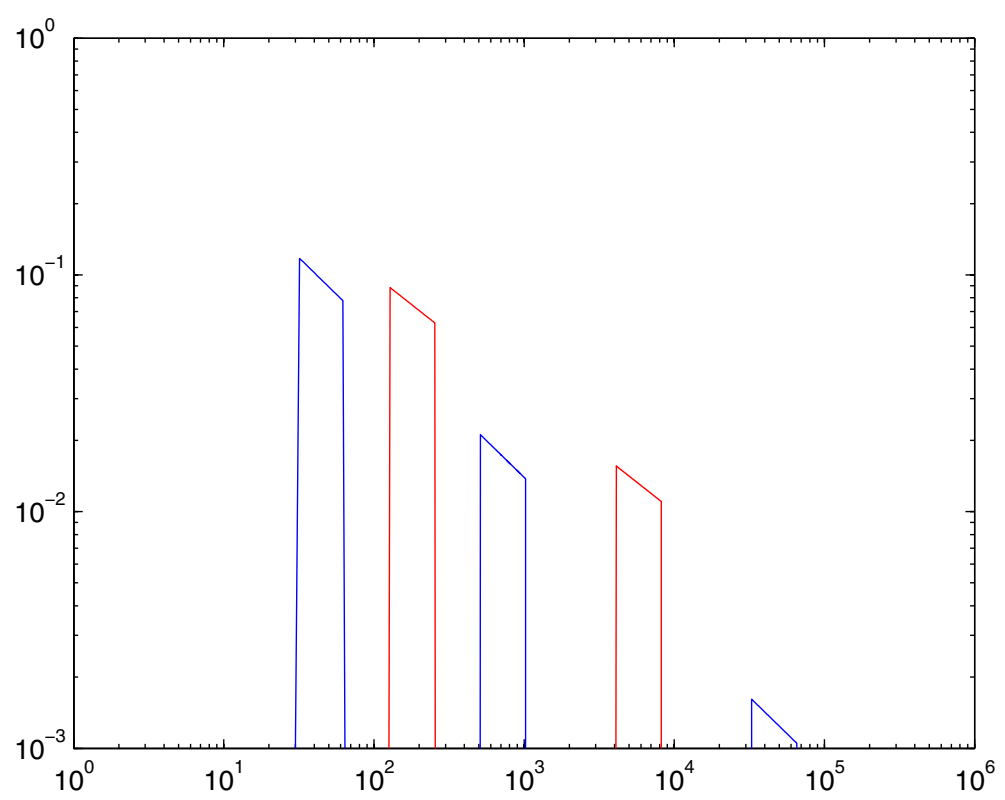

Figure 2. An example of spectral density of LFBM as function of the frequencies.

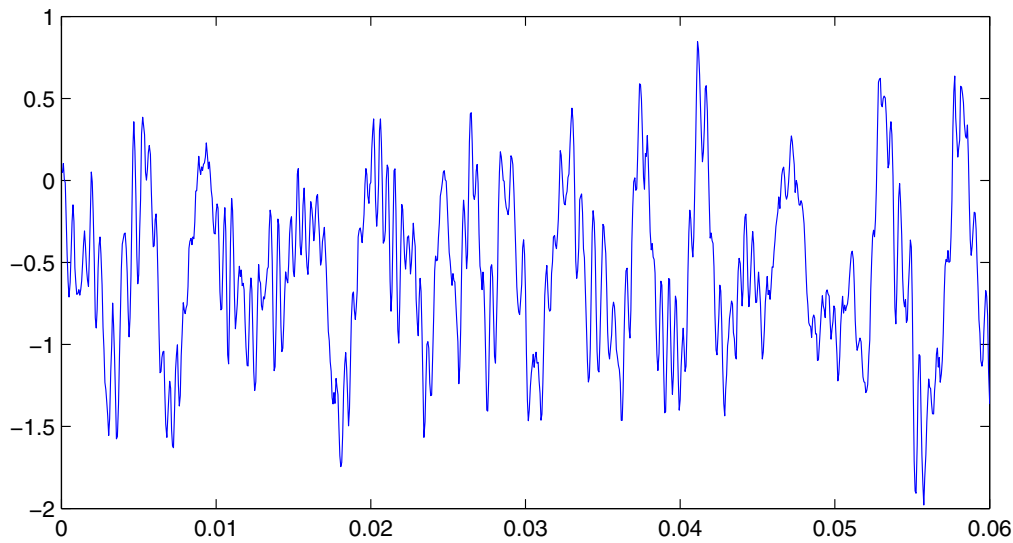

Figure 3. A discretized path $\left(X\left(\Delta_{N}\right), \ldots, X\left(N \Delta_{N}\right)\right)$ with $\underline{H}=0.3$ and $\tau=2$.

\section{Numerical Simulations}

We now illustrate through numerical simulations the multiscale behavior of LFBM. To this end, let $X$ be a LFBM with lower Hurst index $\underline{H}=0.3$ and lacunary index $\tau=2$. A discretized trajectory $\left(X\left(\Delta_{N}\right), \ldots\right.$, $X\left(N \Delta_{N}\right)$ ) is numerically obtained (see Fig. 3) for $N=2000, \Delta_{N}=1 / 2000 \mathrm{using}$ the explicit expression of the covariance and the circulant matrix embedding method.

Recall that this method consists of embedding the covariance matrix of the Gaussian vector $\left(X\left(\Delta_{N}\right), \ldots\right.$, $\left.X\left(N \Delta_{N}\right)\right)$ in a non-negative definite matrix $R$ of size $M \geq 2(N-1)$ which is a circulant one $($ see $[7,12,13,27])$. We also refer to the survey of Bardet et al. (see [3]) for an overview about numerical simulations of Gaussian processes.

Our code is a slight adaptation of the generator of Multi-scale Fractional Brownian Motion furnished to us by Jean-Marc Bardet. 

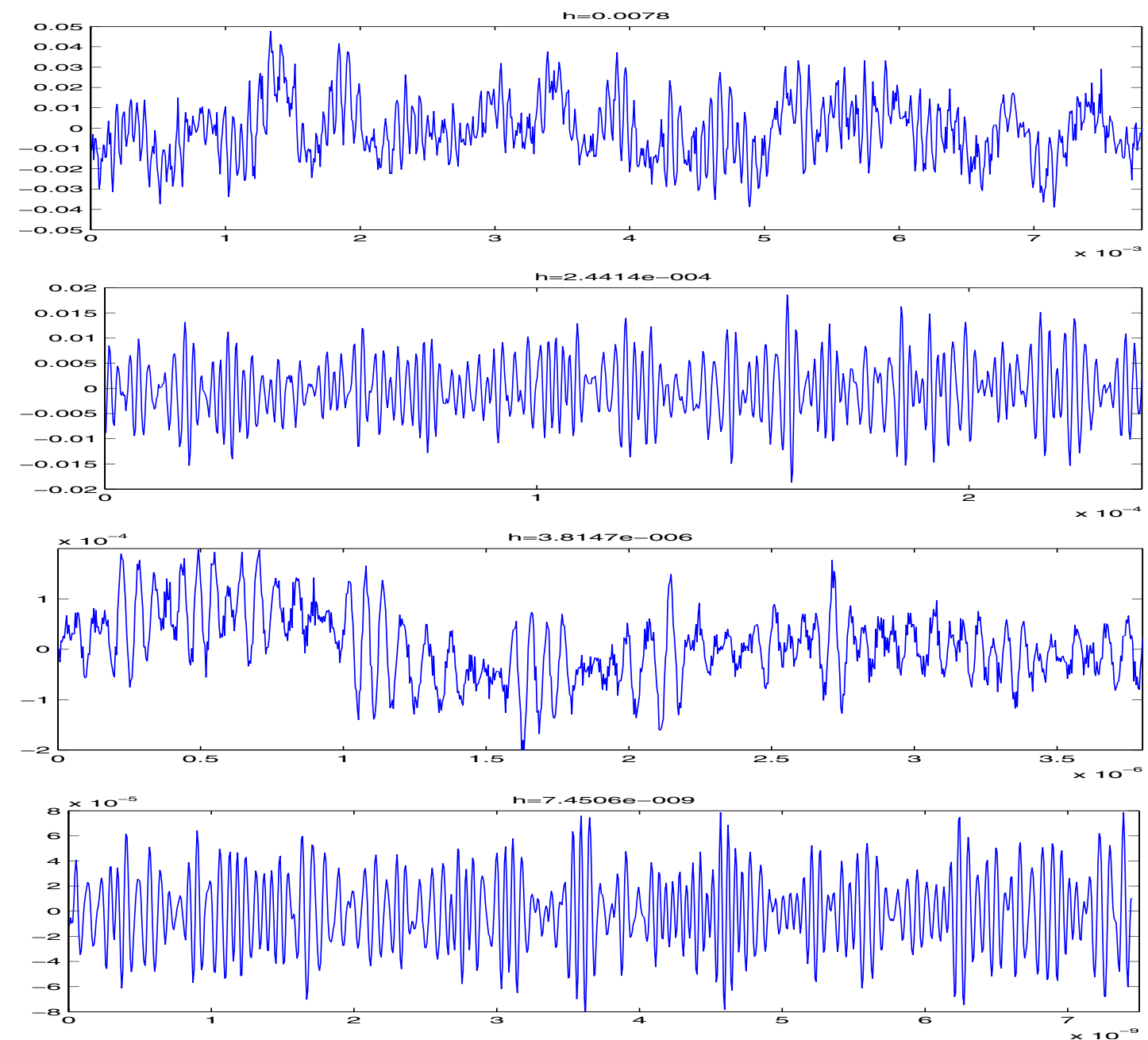

Figure 4. A discretized path of the Gaussian process $x \mapsto X(x+h)-X(x)$ for different values of $h$.

In Figure 4, in order to vizualize the two different behaviors related to the two characteristic family of scales $2^{-j_{n}}$ and $2^{-\ell_{n}}$ involved in the definition of the LFBM $X$, we represent for different values of $h$, a discretized trajectory $\left(X\left(h \Delta_{N}\right), \cdots, X\left(h N \Delta_{N}\right)-X\left(h(N-1) \Delta_{N}\right)\right)$ of the increments of the Gaussian process $X$.

In this case, $N=2000, \underline{H}=0.5, \tau=1.5$ and then $\bar{H}=0.6$. The increments $h$ take the values $2^{-j_{1}}, 2^{-\ell_{1}}, 2^{-j_{2}}, 2^{-\ell_{2}}$ and are linked to the two families of characteristic scales. We see that two regimes begin to appear (compare the first and the third figure and then the second and fourth figure) related to the two different characteristic families of scales and the different Hurst indices $\underline{H}$ and $\bar{H}$.

In this paper, our aim is to state and to prove in a proper way the special self-similarity and sample paths properties satisfied by LFBM. In next Section we investigate the self-similarity properties of LFBM.

\section{Self-similarity properties of LaCunary Fractional Brownian Motion}

The aim of this Section is to state the main result of this paper: LFBM admits several tangent fields at any point (see Sect. 4.2). To this end we need some prerequisites about tangent fields and tangent spaces of a Gaussian field (see Sect. 4.1). 


\subsection{Tangent spaces and tangent fields}

Here, we provide the reader with some material about tangent fields and tangent spaces of a Gaussian field. All that follows can be found with more details in $[15,16]$. The notion of tangent field was initially introduced in [6] by Benassi et al. related to this of local asymptotic self-similarity. In [15] and [16], Falconer generalizes this concept and gives the following general definition:

Definition 4.1. Let $\{X(t)\}_{t \in \mathbb{R}^{d}}$ be a random field with continuous sample paths. The random field $\{Y(t)\}_{t \in \mathbb{R}^{d}}$ vanishing almost surely at $t=0$ is a tangent field at $x_{0}$ to the random field $\{X(t)\}_{t \in \mathbb{R}^{d}}$ if there exist two decreasing sequences of non negative real numbers converging to $0,\left(\rho_{1, n}\right)_{n}$ and $\left(\rho_{2, n}\right)_{n}$ such that

$$
\lim _{n \rightarrow+\infty}\left\{\frac{X\left(x_{0}+\rho_{1, n} t\right)-X\left(x_{0}\right)}{\rho_{2, n}}\right\}_{t \in \mathbb{R}^{d}}=\{Y(t)\}_{t \in \mathbb{R}^{d}}
$$

where lim means the convergence in distribution on the space of continuous functions endowed with the topology of the uniform convergence on compact sets.

In the special case where $\rho_{2, n}=\rho_{1, n}^{H}$ for $H \in(0,1)$, we recover a weak notion of local asymptotic selfsimilarity:

Definition 4.2. Let $\left(\rho_{n}\right)_{n \in \mathbb{N}}$ be a decreasing sequence of non negative real numbers converging to zero. A random field $\{X(x)\}_{x \in \mathbb{R}}$ is locally asymptotically self-similar with exponent $H$ at $x_{0}$ according the family of scales $\left(\rho_{n}\right)_{n \in \mathbb{N}}$ if the random field $\left\{\frac{X\left(x_{0}+\rho_{n} u\right)-X\left(x_{0}\right)}{\rho_{n}^{H}}\right\}_{u \in \mathbb{R}}$ has a non trivial limit when $n$ tends to $+\infty$.

To define formally the concept of uniqueness of tangent field, Falconer needs to use the notion of tangent space. The tangent space of a random field $\{X(t)\}_{t \in \mathbb{R}^{d}}$, written $\operatorname{Tan}\left(X, x_{0}\right)$, is the aggregate of all the tangent fields of $\{X(t)\}_{t \in \mathbb{R}^{d}}$ at $x_{0}$.

One can remark that if $\lambda$ is a positive constant and if $\{Y(t)\}_{t \in \mathbb{R}^{d}}$ is a tangent field to the random field $\{X(t)\}_{t \in \mathbb{R}^{d}}$ then $\{\lambda Y(t)\}_{t \in \mathbb{R}^{d}}$ is also a tangent field to the random field $\{X(t)\}_{t \in \mathbb{R}^{d}}$. The tangent field to $X$ at $x_{0}$ is said to be unique if

$$
\operatorname{Tan}\left(X, x_{0}\right)=\left\{\lambda Y^{\prime}, Y^{\prime} \stackrel{(\mathcal{L})}{=} Y\right\}
$$

for some random field $Y$.

One can also say that $\{Y(t)\}_{t \in \mathbb{R}^{d}}$ is the unique tangent field to the random field $\{X(t)\}_{t \in \mathbb{R}^{d}}$. The random field $X$ admits several tangent fields at $x_{0}$ if there is no uniqueness of the tangent field to $X$ at $x_{0}$.

In the special case of Gaussian fields, Falconer proved the following result (Cor. 3.10 of [16]) which enables to describe the local structure of the tangent space at almost every point:

Theorem 4.3. Let $X$ be a Gaussian field with continuous sample paths. For almost all $x_{0}$ in $\mathbb{R}^{d}$ at which $X$ has a unique tangent field $Y_{x_{0}}$ then the following alternative holds:

(1) Either there exists a d-dimensional Gaussian random variable $Z_{x_{0}}$ such that almost surely,

$$
Y_{x_{0}}(t)=t . Z_{x_{0}}
$$

for all $t$ in $\mathbb{R}^{d}$.

(2) Or there exists $H \in(0,1)$ such that for all $t \neq 0,\left\{Y_{x_{0}}(r t)\right\}_{r \in \mathbb{R}}$ is a constant multiple of index $H$ Fractional Brownian Motion. 
In short, in the case of a Gaussian field admitting at each point a unique tangent field, this tangent field is either 'smooth' or 'fractal' like a FBM. This result comes from two properties of tangent spaces proved in [15,16]: the tangent space of a random field is a.e. shift invariant and always scaling invariant.

One can wonder what kind of behavior can be expected if these assumptions are not satisfied. Using LFBM defined above, we will prove in next section that we can define a Gaussian field admitting several tangent fields at each point and that in that case the tangent fields could be unlike a FBM. Further, we also prove-since we exhibit two tangent fields of LFBM which are not self similar-that in the general case tangent fields are not self-similar.

\subsection{Tangent fields of Lacunary Fractional Brownian Motion}

Now, we state the two central results of this paper concerning the non uniqueness at any point of the tangent field of the LFBM defined by equation (2.6).

Proposition 4.4. (1) The LFBM $\left\{B_{\underline{H}, \tau}(x)\right\}_{x \in \mathbb{R}^{d}}$ defined by equation (2.6) is asymptotically locally selfsimilar with exponent $\underline{H}$ at every point $x_{0}$ according to the family of scales $\left(\rho_{n, 1}\right)_{n \in \mathbb{N}}=\left(2^{-\ell_{n}}\right)_{n}$. Moreover, for all $x_{0} \in \overline{\mathbb{R}}^{d}$

$$
\lim _{n \rightarrow+\infty}\left\{\frac{B_{\underline{H}, \tau}\left(x_{0}+\rho_{n, 1} t\right)-B_{\underline{H}, \tau}\left(x_{0}\right)}{\rho_{n, 1}^{\underline{H}}}\right\}_{t \in \mathbb{R}^{d}}=\left\{\widetilde{B}_{\underline{H}}(t)\right\}_{t \in \mathbb{R}^{d}},
$$

where lim means the convergence in distribution on the space of continuous functions endowed with the topology of the uniform convergence on compact sets and

$$
\widetilde{B}_{\underline{H}}(t)=\int_{\frac{1}{2}<|\xi|<1} \frac{\mathrm{e}^{i t \xi}-1}{|\xi| \underline{H}+\frac{d}{2}} \mathrm{~d} \widehat{W}(\xi) .
$$

(2) The LFBM $\left\{B_{\underline{H}, \tau}(x)\right\}_{x \in \mathbb{R}^{d}}$ defined by equation (2.6) is asymptotically locally self-similar with exponent $\bar{H}$ at every point $x_{0}$ according to the family of scales $\left(\rho_{n, 2}\right)_{n \in \mathbb{N}}=\left(2^{-j_{n}}\right)_{n}$. Moreover, for all $x_{0} \in \mathbb{R}^{d}$

$$
\lim _{n \rightarrow+\infty}\left\{\frac{B_{\underline{H}, \tau}\left(x_{0}+\rho_{n, 2} t\right)-B_{\underline{H}, \tau}\left(x_{0}\right)}{\rho_{n, 2}^{\bar{H}}}\right\}_{t \in \mathbb{R}^{d}}=\left\{\widetilde{B}_{\bar{H}}(t)\right\}_{t \in \mathbb{R}^{d}},
$$

where lim means the convergence in distribution on the space of continuous functions endowed with the topology of the uniform convergence on compact sets and

$$
\widetilde{B}_{\bar{H}}(t)=\int_{\frac{1}{2}<|\xi|<1} \frac{\mathrm{e}^{i t \xi}-1}{|\xi|^{\bar{H}+\frac{d}{2}}} \mathrm{~d} \widehat{W}(\xi) .
$$

Remark 4.5. Remark that the two Gaussian fields $\widetilde{B}_{\underline{H}}$ and $\widetilde{B}_{\bar{H}}$ are not self-similar. The uniqueness assumption is thus necessary to recover the results of Falconer about self-similarity of tangent fields. Since the LFBM admits stationary increments, its tangent fields also admits stationary increments. Hence our model does not yield an example of Gaussian fields with unstationary tangent fields. In [9] the case of a modified version of Generalized Fractional Brownian Motion has been studied. Unfortunately, in this case the tangent fields remains with stationary increments. The question of defining a Gaussian field with unstationary tangent fields remains still open. 
We thus obtain our main result about the structure of tangent space of the LFBM defined by equation (2.6):

Theorem 4.6. For all $x_{0} \in \mathbb{R}^{d}$, the two Gaussian fields $\left\{\widetilde{B}_{\underline{H}}(t)\right\}_{t \in \mathbb{R}^{d}}$ and $\left\{\widetilde{B}_{\bar{H}}(t)\right\}_{t \in \mathbb{R}^{d}}$ defined by equation (4.2) and (4.3) are two tangent fields at $x_{0}$ to the LFBM $\left\{B_{\underline{H}}, \tau(x)\right\}_{x \in \mathbb{R}^{d}}$ defined by equation (2.6). Hence, $\left\{B_{\underline{H}, \tau}(x)\right\}_{x \in \mathbb{R}^{d}}$ admits at every point several tangent fields unlike a FBM.

Let us make some comments about these self-similarity properties of LFBM. Roughly speaking, LFBM 'looks like' the Gaussian field $\left\{\widetilde{B}_{\underline{H}}(t)\right\}_{t \in \mathbb{R}^{d}}$ when being considered at the scales $\left(\rho_{n, 1}\right)_{n \in \mathbb{N}}=\left(2^{-\ell_{n}}\right)_{n}$ whereas it 'looks like' the Gaussian field $\left\{\widetilde{B}_{\bar{H}}(t)\right\}_{t \in \mathbb{R}^{d}}$ when being considered at the scales $\left(\rho_{n, 2}\right)_{n \in \mathbb{N}}=\left(2^{-j_{n}}\right)_{n}$. From the regularity point of view, this non uniform behavior of LFBM according to the different scales is reflected in its sample paths properties studied in next Section.

\section{Sample paths properties of Lacunary Fractional Brownian Motion}

Hereafter we investigate the sample paths properties of LFBM. In Section 5.1, we first give a result about uniform Hölderian regularity of a more general model: ISFBM. Then, in Section 5.2 we focus on the special case of LFBM. We illustrate the non-uniformity in scales of sample paths of LFBM stating our results in terms of uniform irregularity.

\subsection{Regularity of the sample paths of the Infinite Scale Fractional Brownian Motion}

We first study the regularity of the sample paths of LFBM. In fact, our result can be stated for a more general model than LFBM: ISFBM. But before an accurate study of uniform regularity of the sample paths of ISFBM, we first precise the regularity concept we used. This is done in Section 5.1.1.

\subsubsection{Uniform Hölderian regularity}

The usual concept for uniform regularity is uniform Hölderian regularity. Uniform Hölderian regularity of a function can be characterized by its belongness to classical Hölder spaces $\mathcal{C}^{\alpha}\left(\mathbb{R}^{d}\right)$ :

Definition 5.1. A bounded function $f$ belongs to $\mathcal{C}^{\alpha}\left(\mathbb{R}^{d}\right)$ with $\alpha \in(0,1)$ if there exists a real positive number $r_{0}$ and a positive constant $C_{0}$ such that for every scale $0<r \leq r_{0}$,

$$
\sup _{|h| \leq r} \sup _{x \in \mathbb{R}^{d}}|f(x+h)-f(x)| \leq C_{0} r^{\alpha} .
$$

A bounded function $f$ is said to be uniformly Hölder if for some $\varepsilon_{0}>0, f \in \mathcal{C}^{\varepsilon_{0}}\left(\mathbb{R}^{d}\right)$.

In the following, we will be interested in local sample paths properties of ISFBM. To state our result, we need a notion of locally uniform Hölder regularity.

Definition 5.2. The locally bounded function $f$ belongs to $\mathcal{C}_{\text {loc }}^{\alpha}\left(\mathbb{R}^{d}\right)$ with $\alpha \in(0,1)$, if for any $\varphi \in \mathcal{D}\left(\mathbb{R}^{d}\right)$, the function $\varphi f$ belongs to $\mathcal{C}^{\alpha}\left(\mathbb{R}^{d}\right)$. One can define the local uniform Hölder exponent of function $f$ as:

$$
\mathcal{H}_{\text {loc }}(f)=\sup \left\{\alpha>0, f \in \mathcal{C}_{\text {loc }}^{\alpha}\left(\mathbb{R}^{d}\right)\right\} .
$$

Our result of local uniform Hölder regularity of the sample paths of ISFBM is based on wavelets technics. Indeed, discrete wavelet transform and multiresolution analysis are particularly efficient tools to study the uniform Hölderian regularity of a function (see e.g. [23]). Hereafter, we review a result binding the regularity of a function and its wavelet coefficients. We first review some definitions and notations about wavelets (see for more details for example $[22,23])$.

Under some general assumptions, there exists $2^{d}-1$ functions $\left(\psi^{(i)}\right)_{1 \leq i<2^{d}}$, called wavelets, such that $\left\{\psi^{(i)}\left(2^{j} x-j\right): 1 \leq i<2^{d}, k \in \mathbb{Z}^{d}, j \in \mathbb{Z}\right\}$ is an orthogonal basis of $L^{2}\left(\mathbb{R}^{d}\right)$. Any function $f \in L^{2}\left(\mathbb{R}^{d}\right)$ can be decomposed as follows

$$
f(x)=\sum_{j \in \mathbb{Z}} \sum_{k \in \mathbb{Z}^{d}} \sum_{1 \leq i<2^{d}} c_{j, k}^{(i)} \psi^{(i)}\left(2^{j} x-k\right),
$$


where

$$
c_{j, k}^{(i)}=2^{d j} \int_{\mathbb{R}^{d}} f(x) \psi^{(i)}\left(2^{j} x-k\right) \mathrm{d} x .
$$

Let us remark that we do not choose the $L^{2}$ normalization for the wavelets, but rather an $L^{\infty}$ normalization, which is better fitted to the study of Hölder regularity. If $\left(\psi^{(i)}\right)_{i} \in \mathcal{C}^{r}\left(\mathbb{R}^{d}\right)$ and if $\left(\partial^{\alpha} \psi^{(i)}\right)_{i}(|\alpha| \leq r)$ have fast decay, the multiresolution analysis is said to be $r$-smooth. We always suppose that the multiresolution analysis is $r$-smooth with $r>\alpha$.

A dyadic cube of scale $j$ is a cube of the form

$$
\lambda=\left[\frac{k_{1}}{2^{j}}, \frac{k_{1}+1}{2^{j}}\right) \times \cdots \times\left[\frac{k_{d}}{2^{j}}, \frac{k_{d}+1}{2^{j}}\right),
$$

where $k=\left(k_{1}, \ldots, k_{d}\right) \in \mathbb{Z}^{d}$. From now on, wavelets and wavelet coefficients will be indexed with dyadic cubes. Since $i$ takes $2^{d}-1$ values, we can assume that it takes values in $\{0,1\}^{d}-(0, \ldots, 0)$. In the sequel we use the following notations

$$
\begin{aligned}
& \text { - } \lambda=\lambda(i, j, k)=\frac{k}{2^{j}}+\frac{i}{2^{j+\mathrm{T}}}+\left[0, \frac{1}{2^{j+\mathrm{T}}}\right)^{d}, \\
& \text { - } c_{\lambda}=c_{j, k}^{(i)}, \\
& \text { - } \psi_{\lambda}=\psi^{(i)}\left(2^{j}-k\right) .
\end{aligned}
$$

We state the usual wavelet criterium for uniform Hölderian regularity (see [23]).

Theorem 5.3. Let $\alpha \in(0,1), f \in C^{\alpha}\left(\mathbb{R}^{d}\right)$, if and only if there exists $C_{0}>0$ such that

$$
\forall j \geq 0, \sup _{|\lambda|=2^{-j}}\left|c_{\lambda}\right| \leq C_{0} 2^{-\alpha j} .
$$

We now take an interest in the sample paths properties of ISFBM. Next section is devoted to the statement of our regularity result.

\subsubsection{Sample paths Hölderian regularity of Infinite Scale Fractional Brownian Motion}

Now, we consider the local Hölderian regularity properties of the sample paths of ISFBM. When $\sigma \equiv 1$, ISFBM is a special case of GMBM. Therefore we could expect that, as in the case of GMBM (see [1]), the local properties of the sample paths are given by the high frequencies of the harmonizable representation. The following proposition shows that this property is satisfied.

Proposition 5.4. For any integer $j$, let $\tau_{j}=-\frac{\log _{2}\left(\sigma_{j}\right)}{j}$ with $\tau_{j}=+\infty$ if $\sigma_{j}=0$. Set

$$
\underline{H}=\liminf _{j \rightarrow+\infty}\left(H_{j}+\tau_{j}\right)
$$

The local uniform Hölder exponent $\underline{\mathcal{H}}_{\text {loc }}$ of the Infinite Scale Fractional Brownian Motion $\left\{B_{\boldsymbol{H}, \sigma}(x)\right\}_{x \in \mathbb{R}^{d}}$ with Hurst indices $\boldsymbol{H}=\left(H_{j}\right)_{j}$ and amplitudes $\sigma=\left(\sigma_{j}\right)_{j}$ satisfies almost surely

$$
\underline{\mathcal{H}}_{\mathrm{loc}}\left(B_{\boldsymbol{H}, \sigma}\right)=\underline{H} .
$$

This proposition is proved in Section 6.2 using wavelets technics and the wavelet criterion stated in Theorem 5.3 above. Now we focus on the special case of LFBM. Our aim is to understand how the non-uniform behavior of LFBM according to the different scales is reflected in its sample paths properties. To this end we need to complete our study and investigate the irregularity properties of LFBM. 


\subsection{Study of the uniform irregularity of the sample paths in the special case of Lacunary Fractional Brownian Motion}

Here our aim is to illustrate in a relevant way the specific sample paths properties of LFBM using adapted concepts of uniform regularity. In a classical way, we are interested in the behavior of the so-called modulus of continuity of LFBM

$$
r \mapsto \sup _{|h| \leq r} \sup _{x \in K}\left|B_{\underline{H}, \tau}(x+h)-B_{\underline{H}, \tau}(x)\right|
$$

near $r=0$ on any compact $K$. So we are lead to estimate the two following exponents

$$
\underline{\alpha}=\liminf _{r \rightarrow 0} \frac{\log \left(\sup _{|h| \leq r} \sup _{x \in K}\left|B_{\underline{H}, \tau}(x+h)-B_{\underline{H}, \tau}(x)\right|\right)}{\log (r)},
$$

and

$$
\bar{\alpha}=\limsup _{r \rightarrow 0} \frac{\log \left(\sup _{|h| \leq r} \sup _{x \in K}\left|B_{\underline{H}, \tau}(x+h)-B_{\underline{H}, \tau}(x)\right|\right)}{\log (r)}
$$

on any compact $K$.

Remark that $\underline{\alpha}$ coincides with the uniform exponent of the LFBM on the compact $K$ and has already been estimated in Section 5.1. The exponent $\bar{\alpha}$ is related to another concept of uniform regularity than the classical one: weak uniform Hölderian regularity defined in Section 5.2.1. The estimation of $\bar{\alpha}$ (see Sect. 5.2.2) uses the wavelet criterion for weak uniform irregularity stated in Section 5.2.1.

\subsubsection{Alternative definitions of uniform regularity}

We refer to [9] for all the material of this Section. We first introduce the concept of uniform irregularity:

Definition 5.5. A bounded function $f$ is said to be uniformly irregular with exponent $\beta$ if there exists a positive constant $C_{0}$ and a positive real number $r_{0}$ such that for every scale $r$ in $\left(0, r_{0}\right)$

$$
\sup _{|h| \leq r} \sup _{x \in \mathbb{R}^{d}}|f(x+h)-f(x)| \geq C_{0} r^{\beta}
$$

A weak notion for uniform regularity can be associated with the concept of uniform irregularity:

Definition 5.6. A bounded function $f$ is said to be weakly uniformly Hölder of exponent $\beta$ in $(0,1)$ written $f \in \overline{\mathcal{C}}^{\beta}\left(\mathbb{R}^{d}\right)$ if $f \notin I^{\beta}\left(\mathbb{R}^{d}\right)$ i.e. if for any $C>0$, there exists some decreasing sequence $\left(r_{n}\right)_{n \in \mathbb{N}}$ of real positive numbers converging to zero such that

$$
\forall n \in \mathbb{N}, \sup _{|h| \leq r_{n}} \sup _{x \in \mathbb{R}^{d}}|f(x+h)-f(x)| \leq C r_{n}^{\beta}
$$

The uniform irregular exponent of function $f$ is defined as

$$
\mathcal{I}(f)=\inf \left\{\beta>0, f \in I^{\beta}\left(\mathbb{R}^{d}\right)\right\}=\sup \left\{\beta>0, f \in \overline{\mathcal{C}}^{\beta}\left(\mathbb{R}^{d}\right)\right\} .
$$

Remark then that

whereas

$$
\mathcal{I}(f)=\limsup _{r \rightarrow 0} \frac{\log \left(\sup _{|h| \leq r} \sup _{x \in \mathbb{R}^{d}}(|f(x+h)-f(x)|)\right.}{\log (r)} ;
$$

$$
\mathcal{H}(f)=\liminf _{r \rightarrow 0} \frac{\log \left(\sup _{|h| \leq r} \sup _{x \in \mathbb{R}^{d}}(|f(x+h)-f(x)|)\right.}{\log (r)} .
$$


The simultaneous knowledge of the uniform Hölder exponent and the uniform irregular exponent of a bounded function $f$ gives us a lower and an upper bound at $r=0$ of the modulus of continuity of $f, \omega_{f}$, defined as

$$
\omega_{f}(r)=\sup _{|h| \leq r} \sup _{x \in \mathbb{R}^{d}}(|f(x+h)-f(x)|) .
$$

Since we are interested in the local behavior of sample paths, we need the following definitions:

Definition 5.7. The locally bounded function $f$ is said to be locally weak uniformly Hölder with exponent $\beta \in(0,1)$, written $f \in \overline{\mathcal{C}}_{\text {loc }}^{\beta}\left(\mathbb{R}^{d}\right)$, if for any $\varphi \in \mathcal{D}\left(\mathbb{R}^{d}\right)$, the function $\varphi f$ is $\overline{\mathcal{C}}^{\beta}\left(\mathbb{R}^{d}\right)$.

The function $f$ is said to be locally uniformly irregular with exponent exponent $\beta \in(0,1)$, written $f \in$ $I_{\text {loc }}^{\beta}\left(\mathbb{R}^{d}\right)$, if $f \notin \overline{\mathcal{C}}_{\text {loc }}^{\beta}\left(\mathbb{R}^{d}\right)$.

The local irregularity exponent is defined as

$$
\mathcal{I}_{\text {loc }}(f)=\sup \left\{\beta, f \in \overline{\mathcal{C}}_{\text {loc }}^{\beta}\left(\mathbb{R}^{d}\right)\right\} .
$$

To study the irregularity properties of the sample paths of LFBM, we need to use a wavelet criterion for weak uniform Hölder regularity. In [9] is proved the following result

Theorem 5.8. Let $\beta \in(0,1)$.

(1) If $f \in \overline{\mathcal{C}}^{\beta}\left(\mathbb{R}^{d}\right)$ then: for every $C>0$, there exists a strictly increasing sequence of integers $\left(j_{n}\right)_{n} \in \mathbb{N}$ such that:

$$
\left\{\begin{array}{c}
\forall n \geq 0, \forall j \in\left\{j_{n}, \cdots, j_{n+1}-1\right\} \\
\sup _{|\lambda|=2^{-j}}\left(\left|c_{\lambda}\right|\right) \leq C \inf \left(2^{-j_{n} \beta}, 2^{(1-\beta) j_{n+1}} 2^{-j}\right),
\end{array}\right.
$$

(2) conversely, if $f$ is uniformly Hölder and if for any $C>0$, there exists a strictly increasing sequence of integers $\left(j_{n}\right)_{n \in \mathbb{N}}$ such that (5.3) holds, then

$$
\left\{\begin{array}{l}
\forall n \geq 0, \forall j \in\left\{j_{n}, \cdots, j_{n+1}-1\right\} \\
\sup _{x \in \mathbb{R}^{d}} \sup _{|h| \leq 2^{-j}}(|f(x+h)-f(x)|) \leq C_{0} C \inf \left(2^{-j_{n} \beta} j_{n}^{\gamma}, 2^{(1-\beta) j_{n+1}} 2^{-j} j^{\gamma}\right),
\end{array}\right.
$$

where $C_{0}>0$ depends only on multi resolution analysis and $\left(j_{n}\right)_{n}$ denotes the sequence given by (5.3). In particular, $f \in \overline{\mathcal{C}}_{|\log |^{\gamma}}^{\beta}\left(\mathbb{R}^{d}\right)$ for all $\gamma>1$.

Remark 5.9. Remark that, if

$$
\forall j \in \mathbb{N}, \sup _{|\lambda|=2^{-j}}\left(\left|c_{\lambda}\right|\right) \geq C_{0} 2^{-j \beta},
$$

then (5.3) cannot hold and $f$ belongs to $I^{\beta}\left(\mathbb{R}^{d}\right)$. Of course (5.5) is sufficient to prove the uniform irregularity of $f$ but not necessary.

By contraposition of Theorem 5.8, we get a wavelet criterium for uniform irregularity:

Theorem 5.10. Let $\beta \in(0,1)$ and $f$ be a bounded function on $\mathbb{R}^{d}$.

(1) Assume there exists $C_{0}>0$ such that for any integer $j \geq 0$ :

$$
\max \left(\sup _{|\lambda| \leq 2^{-j}}\left(\left|c_{\lambda}\right|\right), 2^{-j} \sup _{|\lambda| \geq 2^{-j}}\left(\frac{\left|c_{\lambda}\right|}{|\lambda|}\right)\right) \geq C_{0} 2^{-j \beta}
$$

then $f \in I^{\beta}\left(\mathbb{R}^{d}\right)$. 
(2) If for any $\gamma>1, f$ is uniformly Hölder and belongs to $I_{|\log | \gamma}^{\beta}\left(\mathbb{R}^{d}\right)$ then there exists $C_{0}>0$ such that for any integer $j \geq 0$ then (5.6) holds.

Now, we will apply these two wavelet criteria to the study of the uniform irregularity of the sample paths of LFBM defined above.

\subsubsection{Smoothness of the sample paths of LFBM}

Let us consider the LFBM defined by equation (2.6). The study of the weak uniform regularity properties will be based on the fact that in short, the large wavelets coefficients of LFBM are located on scales $2^{-\ell_{n}}$ and on the scales $2^{-j_{n}}$ and that, at these scales, the wavelets coefficients satisfy

$$
\sup _{|\lambda|=2^{-\ell_{n}}}\left|c_{\lambda}\right| \approx 2^{-\ell_{n} \underline{H}}, \sup _{|\lambda|=2^{-j_{n}}}\left|c_{\lambda}\right| \approx 2^{-j_{n} \bar{H}}
$$

Thus in Section 6.3 we will prove that,

$$
\mathcal{I}_{\mathrm{loc}}\left(B_{\underline{H}, \tau}\right)=\bar{H}
$$

showing that we are nearly in the case of a lacunary wavelet series of the form

$$
\sum_{n \in \mathbb{N}} \sum_{|\lambda|=2^{-\ell_{n}}} 2^{-\ell_{n}} \underline{H} \psi_{\lambda}+\sum_{n \in \mathbb{N}} \sum_{|\lambda|=2^{-j_{n}}} 2^{-j_{n} \bar{H}} \psi_{\lambda}
$$

The proof is detailed in Section 6.3. Here, we give some heuristic arguments to explain what happens and state our main result about the weak uniform irregularity of LFBM.

The example of LFBM is complicated because there is no lower bound of the wavelets coefficients at every scale of the form

$$
\forall j \geq 0, \sup _{|\lambda|=2^{-j}}\left|c_{\lambda}\right| \geq C_{0} 2^{-j \bar{\alpha}} .
$$

To estimate the uniform irregularity exponent of LFBM, the wavelets criteria recalled in Theorems 5.8 and 5.10 will be needed. Contrary on the uniform Hölder exponent, the uniform irregularity exponent of LFBM will depend on the lacunarity index of the field.

Let us first consider the wavelet series $\sum_{n \in \mathbb{N}|\lambda|=2^{-\ell_{n}}} 2^{-\ell_{n}} \underline{H} \psi_{\lambda}$. Indeed, the wavelets criterium for weak uniform regularity, asserts that the wavelets coefficients at scale $\left(2^{-\ell_{n}}\right)_{n \in \mathbb{N}}$ have an effect on scales below and above the scale $2^{-\ell_{n}}$. This influence is decreasing as far as we get from the scale $2^{-\ell_{n}}$.

This implies that when the band of intermediary scales between the scale $2^{-\ell_{n}}$ is wide, there exists a sequence of intermediary scale $\left(2^{-t_{n}}\right)_{n}$ for which the wavelet coefficients located at the scale $2^{-\ell_{n}}$ have little weight. Thus, the higher the lacunarity index is, the higher the uniform irregularity exponent of this wavelet series and thus of LFBM is.

To prove this, there will be two main difficulties to overcome. Firstly, the sequence $\left(2^{-t_{n}}\right)_{n}$ of intermediate scales has to be defined. This sequence will be defined such that for all $n$, the competitive influence of the large wavelets coefficients located at the scale $2^{-\ell_{n-1}}$ and at the scale $2^{-\ell_{n}}$ are balanced exactly at the scale $2^{-t_{n}}$. Furthermore, the index of uniform irregularity $\bar{\alpha}$ has to be estimated.

We want the uniform irregularity exponent to be larger than an index $\bar{\alpha}$ to estimate. If we suppose that for any $n, t_{n} \leq \ell_{n} \leq t_{n+1}-1$, we will require that,

$$
2^{-\ell_{n} \underline{H}} \leq \inf \left(2^{-t_{n} \bar{\alpha}}, 2^{t_{n+1}(1-\bar{\alpha})-\ell_{n}}\right) .
$$


In the limit case, the relationship above becomes

$$
2^{-\ell_{n} \underline{H}}=2^{-t_{n} \bar{\alpha}}=2^{t_{n+1}(1-\bar{\alpha})-\ell_{n}} .
$$

This means that the influence of wavelets coefficients located at the scale $2^{-\ell_{n}}$ is exactly the same at the intermediary scale just below $2^{-t_{n}}$ and just above $2^{-t_{n+1}}$.

Thus, this leads us to define the following sequence of integer $\left(t_{n}\right)_{n \in \mathbb{N}}$ by,

$$
t_{n}=\left[\frac{\underline{H}}{\bar{\alpha}} \ell_{n}\right]+1
$$

Now, we can express $\bar{\alpha}$ as a function of the lacunarity index $\tau$. By definition of the sequence $\left(t_{n}\right)_{n \in \mathbb{N}}$

$$
\lim _{n} \frac{\ell_{n}}{t_{n}}=\frac{\bar{\alpha}}{\underline{H}} \text { and } \lim _{n} \frac{\ell_{n}}{t_{n+1}}=\frac{1-\bar{\alpha}}{1-\underline{H}} .
$$

Thus

Since $\lim _{n}\left(\ell_{n+1} / \ell_{n}\right)=\tau$, it implies that

$$
\lim _{n} \frac{\ell_{n+1}}{\ell_{n}}=\frac{\bar{\alpha}}{\underline{H}} \times \frac{1-\underline{H}}{1-\bar{\alpha}}
$$

$$
\bar{\alpha}=\frac{\tau \underline{H}}{1-\underline{H}+\tau \underline{H}} .
$$

We then obtain that $\bar{\alpha}=\bar{H}$ and thus the sequence $\left(t_{n}\right)_{n \in \mathbb{N}}$ coincide more or less with the sequence $\left(j_{n}\right)_{n \in \mathbb{N}}$ involved in the definition of LFBM. The uniform irregularity exponent of the wavelet series $\sum_{|\lambda|=2^{-\ell_{n}}} c_{\lambda} \psi_{\lambda}$ should be equal to $\bar{H}$. Moreover, the sequence $\left(j_{n}\right)_{n \in \mathbb{N}}$ has been chosen such that the additional wavelet series $\sum_{|\lambda|=2^{-j_{n}}} c_{\lambda} \psi_{\lambda}$ has not enough large wavelet coefficients to have an influence on the uniform irregularity properties of LFBM whereas it has enough large wavelet coefficients to influence the self-similarity properties of LFBM. This heuristic argument leads us to state the Proposition below proved in Section 6.3:

Proposition 5.11. The sample paths of LFBM defined by equation (2.6) satisfy the following properties:

(1) Almost surely

$$
\mathcal{H}_{\mathrm{loc}}\left(B_{\underline{H}}, \tau\right)=\underline{H} .
$$

Furthermore there exists $C_{1}, C_{2}$ almost surely positive such that for any $n \in \mathbb{N}$

$$
\frac{C_{1} 2^{-\underline{H} \ell_{n}}}{\left|\ell_{n}\right|^{\frac{1}{2}+\varepsilon}} \leq \sup _{\left\{(x, y) \in K^{2},|x-y| \leq 2^{-\ell_{n}}\right\}}\left|B_{\underline{H}, \tau}(x)-B_{\underline{H}, \tau}(y)\right| \leq C_{2} 2^{-\underline{H} \ell_{n}}\left|\ell_{n}\right|^{\tau+\frac{1}{2}} .
$$

(2) Almost surely

$$
\mathcal{I}_{\mathrm{loc}}\left(B_{\underline{H}, \tau}\right)=\bar{H}
$$

Furthermore there exists $C_{3}, C_{4}$ almost surely positive such that for any $n \in \mathbb{N}$

$$
\frac{C_{3} 2^{-j_{n} \bar{H}}}{j_{n}^{\frac{1}{2}+\varepsilon}} \leq \sup _{\left\{(x, y) \in K^{2},|x-y| \leq 2^{-j_{n}}\right\}}\left|B_{\underline{H}, \tau}(x)-B_{\underline{H}, \tau}(y)\right| \leq C_{4} 2^{-j_{n} \bar{H}} j_{n}^{\tau+\frac{1}{2}} .
$$

Next Section is devoted to the proofs of the results stated in the previous sections. 


\section{Proofs}

\subsection{Proof of Proposition 4.4}

The proof of Proposition 4.4 relies on the following lemma:

Lemma 6.1. For all $x, h$ in $\mathbb{R}^{d}$,

$$
\lim _{n_{0} \rightarrow+\infty} \frac{\mathbb{E}\left(\left|B_{\underline{H}, \tau}\left(x+2^{-j_{n_{0}}} h\right)-B_{\underline{H}, \tau}(x)\right|^{2}\right)}{2^{-2 j_{n_{0}} \bar{H}}}=\int_{\frac{1}{2}<|\zeta|<1} \frac{\left|\mathrm{e}^{i h \zeta}-1\right|^{2}}{|\zeta|^{2 \bar{H}+1}} \mathrm{~d} \zeta=\mathbb{E}\left(\left|\widetilde{B}_{\bar{H}}(h)\right|^{2}\right),
$$

and

$$
\lim _{n_{0} \rightarrow+\infty} \frac{\mathbb{E}\left(\left|B_{\underline{H}, \tau}\left(x+2^{-j_{n_{0}, 1}} h\right)-B_{\underline{H}, \tau}(x)\right|^{2}\right)}{2^{-2 j_{n}} \bar{H}}=\int_{\frac{1}{2}<|\xi|<1} \frac{\left|\mathrm{e}^{i h \zeta}-1\right|^{2}}{|\zeta|^{2 \underline{H}}+1} \mathrm{~d} \zeta=\mathbb{E}\left(\left|\widetilde{B}_{\underline{H}}(h)\right|^{2}\right) .
$$

Proof. Since the field $\left\{B_{\underline{H}, \tau}(x)\right\}_{x \in \mathbb{R}^{d}}$ has stationary increments

$$
\begin{aligned}
\mathbb{E}\left(\left|B_{\underline{H}, \tau}\left(x+2^{-j_{n_{0}}} h\right)-B_{\underline{H}, \tau}(x)\right|^{2}\right)-2^{-2 j_{n_{0}} \bar{H}} \int_{\frac{1}{2}<|\xi|<1} \frac{\left|\mathrm{e}^{i h \zeta}-1\right|^{2}}{|\zeta|^{2 \bar{H}+1}} \mathrm{~d} \zeta= & \mathbb{E}\left(\left|B_{\underline{H}, \tau}\left(2^{-j_{n_{0}}} h\right)\right|^{2}\right) \\
& -2^{-2 j_{n_{0}} \bar{H}} \int_{\frac{1}{2}<|\xi|<1} \frac{\left|\mathrm{e}^{i h \zeta}-1\right|^{2}}{|\zeta|^{2 \bar{H}+1} \mathrm{~d} \zeta .}
\end{aligned}
$$

Moreover, performing the change of variables $\zeta=2^{-j_{n_{0}}} \xi$ in equation (2.6) leads to

$$
\begin{aligned}
\mathbb{E}\left(\mid B_{\underline{H}, \tau}\left(x+2^{-j_{n_{0}}} h\right)-\right. & \left.\left.B_{\underline{H}, \tau}(x)\right|^{2}\right)-2^{-2 j_{n_{0}} \bar{H}} \int_{\frac{1}{2}<|\xi|<1} \frac{\left|\mathrm{e}^{i h \zeta}-1\right|^{2}}{|\zeta|^{2 \bar{H}+1}} \mathrm{~d} \zeta=\sum_{n=0}^{+\infty} 2^{-2 j_{n_{0}} \underline{H}} \\
& \times \int_{2^{-j_{n_{0}}} D_{\ell_{n}}} \frac{\left|\mathrm{e}^{i h \zeta}-1\right|^{2}}{|\zeta|^{2 \underline{H}+1}} \mathrm{~d} \zeta+\sum_{n=0}^{+\infty} 2^{-2 j_{n_{0}} \bar{H}} \int_{2^{-j_{n_{0}}}{ }_{D_{j_{n}}}} \frac{\left|\mathrm{e}^{i h \zeta}-1\right|^{2}}{|\zeta|^{2 \bar{H}+1}} \mathrm{~d} \zeta-\int_{\frac{1}{2}<|\xi|<1} \frac{\left|\mathrm{e}^{i h \zeta}-1\right|^{2}}{|\zeta|^{2 \bar{H}+1}} \mathrm{~d} \zeta .
\end{aligned}
$$

Hence

$$
\begin{aligned}
& \mathbb{E}\left(\left|B_{\underline{H}, \tau}\left(x+2^{-j_{n_{0}}} h\right)-B_{\underline{H}, \tau}(x)\right|^{2}\right)-2^{-2 j_{n_{0}} \bar{H}}= \\
& \sum_{n=0}^{n_{0}-1} 2^{-2 j_{n_{0}} \underline{H}} \int_{2^{-j_{n_{0}}}} \frac{\left|\mathrm{e}_{D_{\ell_{n}}} \underline{\zeta \zeta}-1\right|^{2}}{|\zeta|^{2} \underline{H}+1} \mathrm{~d} \zeta+\sum_{n=n_{0}}^{+\infty} 2^{-2 j_{n_{0}} \underline{H}} \int_{2^{-j_{n_{0}}}} \frac{\left|\mathrm{e}_{D_{\ell_{n}}}^{i h \zeta}-1\right|^{2}}{|\zeta|^{2 \underline{H}+1}} \mathrm{~d} \zeta \\
& +\sum_{n=0}^{n_{0}-1} 2^{-2 j_{n_{0}} \bar{H}} \int_{2^{-j_{n_{0}}}} \frac{\left|\mathrm{e}^{i h \zeta}-1\right|^{2}}{|\zeta|^{2 \bar{H}+1}} \mathrm{~d} \zeta+\sum_{n=n_{0}+1}^{+\infty} 2^{-2 j_{n_{0}} \bar{H}} \int_{2^{-j_{n_{0}}}} \frac{\left|\mathrm{e}^{i h \zeta}-1\right|^{2}}{|\zeta|^{2 \bar{H}+1}} \mathrm{~d} \zeta .
\end{aligned}
$$

We will give upper bounds for each sum above. We bound the first sum in the following way:

$$
\begin{aligned}
& \sum_{n=0}^{n_{0}-1} 2^{-j_{n_{0}} \underline{H}} \int_{2^{-j_{n_{0}}}} \frac{\mid \mathrm{e}_{\ell_{\ell_{n}}} \frac{i h \zeta}{|\zeta|^{2}}}{|\zeta|^{2} \underline{H}+1} \mathrm{~d} \zeta \leq \sum_{n=0}^{n_{0}-1} 2^{-2 j_{n_{0}} \underline{H}} \int_{2^{-j_{n_{0}}} \int_{D_{\ell_{n}}}}|h|^{2}|\zeta|^{1-2 \underline{H}} \mathrm{~d} \zeta \\
& \leq|h|^{2} 2^{-2 j_{n_{0}}} \underline{\underline{H}} \sum_{n=0}^{n_{0}-1} 2^{2\left(\ell_{n}-j_{n_{0}}\right)(1-\underline{H})} \\
& \leq|h|^{2} 2^{-2 j_{n_{0}} \underline{H}} 2^{n=0} 2^{2\left(j_{n_{0}-1,1}-j_{n_{0}}\right)(1-\underline{H})} \\
& \leq|h|^{2} 2^{-2 j_{n_{0}}} 2^{2 j_{n_{0}}(1-\bar{H})-\alpha_{1} \log \left(j_{n_{0}}\right)} \\
& \leq|h|^{2} \frac{2^{-2 j_{n_{0}}(1-\bar{H})} 2^{-2 \bar{H} j_{n_{0}}}}{j_{n_{0}}^{\alpha_{1}}}
\end{aligned}
$$

for some $\alpha_{1}>0$ depending on $\gamma$ and $\bar{H}$. 
We then bound the second sum:

$$
\begin{aligned}
& \sum_{n=n_{0}}^{+\infty} 2^{-2 j_{n_{0}} \underline{H}} \int_{2^{-j_{n_{0}}} \int_{D_{\ell_{n}}} \frac{\left|\mathrm{e}^{i h \zeta}-1\right|^{2}}{|\zeta|^{2 \underline{H}}+1} \mathrm{~d} \zeta} \leq 2^{-2 j_{n_{0}} \underline{H}} \sum_{n_{0}}^{+\infty} \int_{2^{-j_{n_{0}}}} \frac{\mathrm{d} \zeta}{|\zeta|^{2 \underline{H}+1}} \\
& \leq 2^{-2 j_{n_{0}} \underline{H}} \sum_{n=n_{0}}^{+\infty} \int_{2^{-j_{n_{0}}}} \frac{\mathrm{d} \zeta}{|\zeta|^{2} \underline{H}+1} \\
& \leq 2^{-2 j_{\ell_{0}}} \underline{H} \sum_{n=n_{0}}^{+\infty} 2^{-2\left(\ell_{n}-j_{n_{0}}\right) \underline{H}} \\
& \leq 2^{-2 \ell_{n_{0}} \underline{H}}=\frac{2^{-2 j_{n_{0}} \bar{H} \lambda}}{j_{n_{0}}^{\bar{H} \gamma}}
\end{aligned}
$$

using the definition of $\ell_{n_{0}}$.

The third sum can be bounded in the same way as the first one

$$
\begin{aligned}
\sum_{n=0}^{n_{0}-1} 2^{-2 j_{n_{0}} \bar{H}} \int_{2^{-j_{n_{0}}} \int_{D_{j_{n}}} \frac{\left|\mathrm{e}^{i h \zeta}-1\right|^{2}}{|\zeta|^{2 \bar{H}+1}} \mathrm{~d} \zeta} & 2^{-2 j_{n_{0}} \bar{H}} \sum_{n=0}^{n_{0}-1} 2^{2\left(j_{n}-j_{n_{0}}\right)(1-\bar{H})} \\
& \leq 2^{-2 j_{n_{0}} \bar{H}} 2^{2\left(j_{n_{0}-1}-j_{n_{0}}\right)(1-\bar{H})} \\
& \leq 2^{-2 j_{n_{0}} \bar{H}} j_{n_{0}}^{2(1-\bar{H})} 2^{-2 j_{n_{0}}\left(1-\frac{1}{\beta}\right)(1-\bar{H})}
\end{aligned}
$$

using (2.5).

The fourth sum can be bounded in the same way as the second one,

$$
\begin{aligned}
\sum_{n=n_{0}+1}^{+\infty} 2^{-2 j_{n_{0}} \bar{H}} \int_{2^{-j_{n}}} \frac{\left|\mathrm{e}^{i h \zeta}-1\right|^{2}}{|\zeta|^{2 \bar{H}+1}} \mathrm{~d} \zeta & \leq 2^{-2 j_{n_{0}} \bar{H}} \sum_{n=n_{0}+1}^{+\infty} 2^{-2\left(j_{n}-j_{n_{0}}\right) \bar{H}} \\
& \leq 2^{-2 j_{n_{0}} \bar{H}} 2^{-2\left(j_{n_{0}+1}-j_{n_{0}}\right) \bar{H}} \\
& \leq 2^{-2 j_{n_{0}} \bar{H}} j_{n_{0}}^{\gamma} 2^{-2 j_{n_{0}}(\beta-1) \bar{H}} .
\end{aligned}
$$

Thus we proved (6.1). The proof of (6.2) is similar.

The above lemma yields the convergence of the finite dimensional distribution of the field $\left\{\frac{B_{\underline{H}, \tau}\left(x+2^{-j_{n}} u\right)-B_{\underline{H}, \tau}(x)}{2^{-j_{n} \bar{H}}}\right\}_{u \in \mathbb{R}^{d}}$ to those of $\left\{\widetilde{B}_{\bar{H}}(t)\right\}_{t \in \mathbb{R}^{d}}$. We also proved the convergence of the finite dimensional distribution of the field $\left\{\frac{B_{\underline{H}, \tau}\left(x+2^{-\ell_{n}} u\right)-B_{\underline{H}, \tau}(x)}{2^{-\ell_{n} \underline{H}}}\right\}_{u \in \mathbb{R}^{d}}$ to those of $\left\{\widetilde{B}_{\underline{H}}(t)\right\}_{t \in \mathbb{R}^{d}}$.

In order to prove the convergence in distribution for the topology of the uniform convergence on compact sets the following tightness result is required (see for e.g. the Kolmogorov criterium recalled in [19]):

Proposition 6.2. Let $\left(Z_{n}\right)_{n \in \mathbb{N}}$ a sequence of continuous random fields on $\mathbb{R}^{d}$ vanishing at the origin. Suppose that for all $T>0$,

$$
\forall(s, t) \in[-T, T]^{d}, \sup _{n \in \mathbb{N}} \mathbb{E}\left(\left|Z_{n}(t)-Z_{n}(s)\right|^{\alpha}\right) \leq C_{0}(T)|t-s|^{1+\beta},
$$

for some positive constant $C_{0}(T)$ which may depend on $T$ and $\alpha, \beta$ which are universal positive constants. If, moreover, for any compact $K$ the finite dimensional distributions of sequence $\left(Z_{n}\right)_{n \in \mathbb{N}}$ converge on $K$ then the convergence is in distribution on $K$. 
Using Proposition 6.2 above and a method similar to the prove of Lemma 6.1, we apply this tightness criterium to the family,

$$
\left\{Z_{n}^{1}(u)\right\}_{u \in \mathbb{R}^{d}}=\left\{\frac{B_{\underline{H}, \tau}\left(x+2^{-j_{n}} u\right)-B_{\underline{H}, \tau}(x)}{2^{-j_{n} \bar{H}}}\right\}_{u \in \mathbb{R}^{d}}
$$

and to the family

$$
\left\{Z_{n}^{2}(u)\right\}_{u \in \mathbb{R}^{d}}=\left\{\frac{B_{\underline{H}, \tau}\left(x+2^{-\ell_{n}} u\right)-B_{\underline{H}, \tau}(x)}{2^{-\ell_{n} \underline{H}}}\right\}_{u \in \mathbb{R}^{d}} .
$$

So we can conclude.

\subsection{Proof of Proposition $\mathbf{5 . 4}$}

The proof uses several technics introduced in [8]; (5.2) will be obtained through a serie expansion of $B_{\mathbf{H}, \sigma}$ in the orthonormal basis of $L^{2}\left(\mathbb{R}^{d}\right)$ given by the Lemarié-Meyer wavelets (see [23]).

The Proof of Proposition 5.4 relies on a key lemma that we state and prove in next section.

6.2.1. Estimates on the wavelet coefficients of the field

Lemme 6.3. Let $j \geq 1,\left(k, k^{\prime}\right) \in\left(\mathbb{Z}^{d}\right)^{2}$.

(1) The following estimates of the covariance of the wavelets coefficients of the field $\left\{B_{H, \sigma}(x)\right\}_{x \in \mathbb{R}^{d}}$ hold

$$
\exists C_{0}>0,\left|\mathbb{E}\left(c_{j, k} c_{j, k^{\prime}}\right)\right| \leq C_{0} \frac{\max \left(\sigma_{j}^{2} 2^{-j H_{j}}, \sigma_{j+1}^{2} 2^{-j H_{j+1}}, \sigma_{j+2}^{2} 2^{-j H_{j+2}}\right)}{1+\left|k-k^{\prime}\right|} .
$$

(2) If $k=k^{\prime}$ then

$$
\exists C_{1}, C_{2}>0 C_{1} \max _{\ell \in\{j, j+1, j+2\}}\left(\sigma_{\ell}^{2} 2^{-2 j H_{\ell}}\right) \leq \mathbb{E}\left(c_{j, k}^{2}\right) \leq C_{2} \max _{\ell \in\{j, j+1, j+2\}}\left(\sigma_{\ell}^{2} 2^{-2 j H_{\ell}}\right) .
$$

Proof.

(1) We use the Meyer wavelet basis which satisfies for any $i, \operatorname{supp}\left(\widehat{\psi}^{(i)}\right) \subset[-4 \pi / 3,-2 \pi / 3] \cup[2 \pi / 3,4 \pi / 3]$. The proof is based on the harmonisable representation of wavelet coefficients of ISFBM. For any Gaussian field with stationary increments admitting a spectral density $f$, one has

$$
\forall j \geq 1, \forall k \in \mathbb{Z}^{d}, c_{j, k}^{i}=\int_{\mathbb{R}^{d}} \mathrm{e}^{i 2^{-j} k \xi} \overline{\widehat{\psi}^{(i)}}\left(2^{-j} \xi\right) f(\xi)^{1 / 2} \mathrm{~d} \widehat{W}(\xi) .
$$

Hence, in the special case of ISFBM, one deduces that

$$
\forall j \geq 1, \forall k \in \mathbb{Z}^{d}, c_{j, k}^{i}=\sum_{\ell=0}^{+\infty} \sigma_{\ell} \int_{D_{\ell}} \frac{\mathrm{e}^{i k 2^{-j}} \bar{\xi} \widehat{\bar{\psi}}^{(i)}\left(2^{-j} \xi\right)}{|\xi|^{H_{\ell}+d / 2}} \mathrm{~d} \widehat{W}(\xi) .
$$

Then for any $i \in\left\{1, \ldots, 2^{d}-1\right\}, j \geq 1$ and any $\left(k, k^{\prime}\right) \in\left(\mathbb{Z}^{d}\right)^{2}$

$$
\mathbb{E}\left(c_{j, k}^{i} c_{j, k^{\prime}}^{i}\right)=\sum_{\ell=0}^{+\infty} \sigma_{\ell}^{2} \int_{D_{\ell}} \frac{\mathrm{e}^{i 2^{-j}\left(k-k^{\prime}\right) \xi}\left|\widehat{\psi}^{(i)}\left(2^{-j} \xi\right)\right|^{2}}{|\xi|^{2 H_{\ell}+d}} \mathrm{~d} \xi .
$$


Perform the change of variable $\zeta=2^{-j} \xi$ :

$$
\begin{aligned}
\mathbb{E}\left(c_{j, k}^{i} c_{j, k^{\prime}}^{i}\right)= & \sigma_{j}^{2} 2^{-2 j H_{j}} \int_{1<|\zeta|<2} \frac{\mathrm{e}^{i\left(k-k^{\prime}\right) \zeta\left|\widehat{\psi}^{(i)}(\zeta)\right|^{2}}}{|\zeta|^{2 H_{j}+d}} \mathrm{~d} \zeta \\
& +\sigma_{j+1}^{2} 2^{-2 j H_{j+1}} \int_{2<|\zeta|<4} \frac{\mathrm{e}^{i\left(k-k^{\prime}\right) \zeta}\left|\widehat{\psi}^{(i)}(\zeta)\right|^{2}}{|\zeta|^{2 H_{j+1}+d}} \mathrm{~d} \zeta \\
& +\sigma_{j+2}^{2} 2^{-2 j H_{j+2}} \int_{4<|\zeta|<8} \frac{\mathrm{e}^{i\left(k-k^{\prime}\right) \zeta}\left|\widehat{\psi}^{(i)}(\zeta)\right|^{2}}{|\zeta|^{2 H_{j+2}+d}} \mathrm{~d} \zeta .
\end{aligned}
$$

Let $j$ a given integer and $p \in\{1,2,3\}$, we define $F_{j}^{p}$ as

$$
F_{j}^{p}(x)=\int_{2^{p}<|\zeta|<2^{p+1}} \frac{\mathrm{e}^{i x \zeta}\left|\widehat{\psi}^{(i)}(\zeta)\right|^{2}}{|\zeta|^{2 H_{j}+d}} \mathrm{~d} \zeta
$$

We want to bound

Remark that

$$
\sup _{x \in \mathbb{R}^{d}}\left((1+|x|)\left|F_{j}^{p}(x)\right|\right)
$$

$$
\left\|F_{j}^{p}\right\|_{L^{\infty}\left(\mathbb{R}^{d}\right)} \leq \int_{2^{p}<|\zeta|<2^{p+1}} \frac{|\widehat{\psi}(\zeta)|^{2}}{|\zeta|^{2 H_{j}+d}} \mathrm{~d} \zeta \leq \int_{1<|\zeta|<8} \frac{|\widehat{\psi}(\zeta)|^{2}}{|\zeta|^{2} \underline{H}+d} \mathrm{~d} \zeta=C(\underline{H}) .
$$

Furthermore, for all $\ell$ and $p$ (perform an integral by parts):

$$
x_{\ell} F_{j}^{p}=\mathrm{e}^{i x_{\ell} 2^{p+1}} \frac{\left|\widehat{\psi}\left(2^{p+1}\right)\right|^{2}}{\left|2^{p+1}\right|^{2 H_{j}+d}}-\mathrm{e}^{i x_{\ell} 2^{p}} \frac{\left|\widehat{\psi}\left(2^{p}\right)\right|^{2}}{\left|2^{p}\right|^{2 H_{j}+d}}-\int_{2^{p}<|\zeta|<2^{p+1}} \mathrm{e}^{i x \zeta} \frac{\partial^{\ell}\left(\left|\widehat{\psi}^{(i)}(\zeta)\right|^{2} /|\zeta|^{2 H_{j}+d}\right)}{\partial \zeta_{\ell}} \mathrm{d} \zeta
$$

which can be bounded in a similar way than $\left\|F_{j}^{p}\right\|_{L^{\infty}\left(\mathbb{R}^{d}\right)}$ since $0 \notin \operatorname{supp}(\widehat{\psi})$.

If we set $x=k-k^{\prime}$, we thus obtain the required result.

(2) If $k=k^{\prime}$ then,

$$
\begin{aligned}
\mathbb{E}\left(\left|c_{j, k}^{i}\right|^{2}\right)= & \sigma_{j}^{2} 2^{-2 j H_{j}} \int_{1<|\zeta|<2} \frac{|\widehat{\psi}(\zeta)|^{2}}{|\zeta|^{2 H_{j}+d}} \mathrm{~d} \zeta+\sigma_{j+1}^{2} 2^{-2 j H_{j+1}} \int_{2<|\zeta|<4} \frac{|\widehat{\psi}(\zeta)|^{2}}{|\zeta|^{2 H_{j+1}+d}} \mathrm{~d} \zeta \\
& +\sigma_{j+2}^{2} 2^{-2 j H_{j+2}} \int_{4<|\zeta|<8} \frac{|\widehat{\psi}(\zeta)|^{2}}{|\zeta|^{2 H_{j+2}+d}} \mathrm{~d} \zeta \\
\geq & \sigma_{j}^{2} 2^{-2 j H_{j}} \int_{1<|\zeta|<2} \frac{|\widehat{\psi}(\zeta)|^{2}}{|\zeta|^{2 \bar{H}+d}} \mathrm{~d} \zeta+\sigma_{j+1}^{2} 2^{-2 j H_{j+1}} \int_{2<|\zeta|<4} \frac{|\widehat{\psi}(\zeta)|^{2}}{|\zeta|^{2 \bar{H}+d}} \mathrm{~d} \zeta \\
& +\sigma_{j+2}^{2} 2^{-2 j H_{j+2}} \int_{4<|\zeta|<8} \frac{|\widehat{\psi}(\zeta)|^{2}}{|\zeta|^{2} \bar{H}+d} \mathrm{~d} \zeta .
\end{aligned}
$$

The result follows. 


\subsubsection{Determination of the local uniform Hölder exponent}

Let

$$
B_{\mathbf{H}, \sigma}^{1}(x)=\sum_{j \geq 0} \sum_{|k|<j 2^{j d}} c_{j, k}^{i}(\omega) \psi_{j, k}^{i}(x)
$$

and

$$
B_{\mathbf{H}, \sigma}^{2}(x)=\sum_{k \in \mathbb{Z}^{d}} C_{k}(\omega) \phi_{k}(x)+\sum_{j \geq 0} \sum_{|k|>j 2^{j d}} c_{j, k}^{i}(\omega) \psi_{j, k}^{i}(x) .
$$

We will focus on the estimate of the uniform regularity exponent of field $\left\{B_{\mathbf{H}, \sigma}^{1}(x)\right\}_{x \in \mathbb{R}^{d}}$.

We first prove that

$$
\underline{H} \geq \mathcal{H}_{\mathrm{loc}}\left(B_{\mathbf{H}, \sigma}^{1}\right) .
$$

For any integer $j$, let us set $\tau_{j}=-\frac{\log _{2}\left(\sigma_{j}\right)}{j}$ with $\tau_{j}=+\infty$ if $\sigma_{j}=0$.

By assumption, for any positive real $\varepsilon$, there exists an integer $j_{0}$ such that,

$$
\forall j \geq j_{0}, H_{j}+\tau_{j} \geq \underline{H}-\varepsilon .
$$

Then, by a classical lemma (see Lem. 3 of [24]) and Lemma 6.3 we deduce that there exists a almost surely positive constant $C_{0}(\omega)$ and an integer $j_{0}(\omega)$ such that for any $j \geq j_{0}(\omega)$ and any $k \in \mathbb{Z}^{d}$

$$
\begin{aligned}
\left|c_{j, k}^{i}\right| & \leq C_{0} \sqrt{\log (k)} \sqrt{\log (j)} \max \left(2^{-j\left(H_{j}+\tau_{j}\right)}, 2^{-j\left(H_{j+1}+\tau_{j+1}\right)}, 2^{-j\left(H_{j+2}+\tau_{j+2}\right)}\right) \\
& \leq C_{0} j \sqrt{\log (j)} \max \left(2^{-j\left(H_{j}+\tau_{j}\right)}, 2^{-j\left(H_{j+1}+\tau_{j+1}\right)}, 2^{-j\left(H_{j+2}+\tau_{j+2}\right)}\right) \\
& \leq C_{0} j \sqrt{\log (j)} 2^{-j(\underline{H}-\varepsilon)}
\end{aligned}
$$

Hence, if $|k| \leq j 2^{j d}$

$$
\begin{aligned}
\left|c_{j, k}^{i}\right| & \leq C_{0} j d \sqrt{\log (j)} \max \left(2^{-j\left(H_{j}+\tau_{j}\right)}, 2^{-j\left(H_{j+1}+\tau_{j+1}\right)}, 2^{-j\left(H_{j+2}+\tau_{j+2}\right)}\right) \\
& \leq C_{0} j \sqrt{\log (j)} 2^{-j(\underline{H}-\varepsilon)} .
\end{aligned}
$$

Thus, by the usual wavelet criterium for uniform Hölder regularity (see Thm. 5.3 above) we deduce the required upper bound of uniform Hölder exponent of the random field $B_{\mathbf{H}, \sigma}^{1}$.

Theorem II.7 of [8] applied to the sequence $\left(g_{n, k, i}\right)=\frac{c_{\ell_{n}, k}^{i}}{\mathbb{E}\left(\left|c_{\ell_{n}, k}^{i}\right|^{2}\right)^{\frac{1}{2}}}$ yields the converse inequality

$$
\underline{H} \leq \mathcal{H}_{\mathrm{loc}}\left(B_{\mathbf{H}, \sigma}^{1}\right)
$$

by using the first point of Lemma 6.3. The conclusion is then straightforward using the following lemma:

Lemma 6.4. Almost surely, the sample paths of the field $\left\{B_{\boldsymbol{H}, \sigma}^{2}(x)\right\}_{x \in \mathbb{R}^{d}}$ are $\mathcal{C}_{\text {loc }}^{H^{\prime}}\left(\mathbb{R}^{d}\right)$ for any

$$
0<\bar{H}<H^{\prime}<1
$$

Then, the uniform regularity and irregularity exponents of the sample paths of ISFBM are those of the field $\left\{B_{H, \sigma}^{1}(x)\right\}_{x \in \mathbb{R}^{d}}$.

Proof. Let $0<\bar{H}<H^{\prime}<1, \varepsilon>0$ and $\varphi \in \mathcal{D}\left(\mathbb{R}^{d}\right)$. We may assume that $\operatorname{supp}(\varphi) \subset B_{d}(0,1)=\{x,|x| \leq 1\}$ and $0 \leq \varphi \leq 1$. We denote by $Y$ the random field $\varphi B_{\mathbf{H}, \sigma}^{2}$. We want to give an upper bound of $|Y(x+h)-Y(x)|$ for any given $x, h$ in $B_{d}(0,1)$.

Here $\Psi_{j, k}^{i}, \Phi_{k}$ will denote respectively $\varphi \psi_{j, k}^{i}$ and $\varphi \phi_{k}$. Remark that if

$$
f_{j, k}=\Psi_{j, k}\left(2^{-j} \cdot\right)
$$


then

$$
\left|f_{j, k}\right| \leq \psi(\cdot-k)
$$

and

Using (6.4)

$$
\Psi_{j, k}(x+h)-\Psi_{j, k}(x)=f_{j, k}\left(2^{j} x+2^{j} h\right)-f_{j, k}\left(2^{j} x\right) .
$$

$$
\begin{aligned}
|Y(x+h)-Y(x)|= & \left|\sum_{j=1}^{+\infty} \sum_{|k|>j 2^{j d}} c_{j, k}^{i}\left(f_{j, k}\left(2^{j} x+2^{j} h\right)-f_{j, k}\left(2^{j} x\right)\right)+\sum_{k \in \mathbb{Z}^{d}} C_{k}\left(\Phi_{k}(x+h)-\Phi_{k}(x)\right)\right| \\
& \leq \sum_{j=1}^{+\infty} \sup _{y \in\left[2^{j} x, 2^{j} x+2^{j}\right.} \sum_{|\gamma|=1|k|>j 2^{j d}} \frac{2^{-j(\underline{H}-\varepsilon)} \log (2+|k|) \log (2+j)\left|2^{j} h\right|}{(1+|y-k|)^{M}} \\
& +|h| \sup _{y \in[x, x+h]} \sum_{k \in \mathbb{Z}^{d}}\left(\frac{\sqrt{\log (2+|k|)}}{\left(1+|y-k|^{M}\right)}\right) \mid
\end{aligned}
$$

using (6.6) and the fast decay of $\phi$ and $\psi$.

Then

$$
|Y(x+h)-Y(x)| \leq|h|\left(\sum_{j=1}^{+\infty}\left(\frac{C 2^{j(1-\underline{H}-\varepsilon)} j \log (2+j)}{\left(1+j 2^{j}\right)^{M}}\right)+C\right)
$$

The lemma follows if we choose $M$ suficiently large.

\subsection{Proof of Proposition 5.11}

We will use the wavelets criterium recalled in Section 5. By definition of the two sequences $\left(j_{n}\right)_{n},\left(\ell_{n}\right)_{n}$ and by Lemma 6.3:

For any integer $n$, and all $j \in\left\{j_{n}, \ldots, j_{n+1}-1\right\}$

$$
\left\{\begin{array}{l}
\max _{j \in\left\{\ell_{n}-2, \cdots, \ell_{n}+2\right\}}\left(\mathbb{E}\left(\left|c_{j, k}^{i}\right|^{2}\right)\right) \leq C_{0} 2^{-2 \ell_{n} \underline{H}}, \\
\max _{j \in\left\{j_{n}, j_{n}+1, j_{n}+2\right\}}\left(\mathbb{E}\left(\left|c_{j, k}^{i}\right|^{2}\right)\right) \leq C_{0} 2^{-2 j_{n} \bar{H}}, \\
\max _{j \in\left\{j_{n+1}-2, j_{n+1}-1\right\}}\left(\mathbb{E}\left(\left|c_{j, k}^{i}\right|^{2}\right)\right) \leq C_{0} 2^{-2 j_{n+1} \bar{H}}
\end{array}\right.
$$

and if $j \notin\left\{\ell_{n}-2, \cdots, \ell_{n+2}\right\} \cup\left\{j_{n}, j_{n}+1, j_{n}+2\right\} \cup\left\{j_{n+1}-2, j_{n+1}-1\right\}, \mathbb{E}\left(\left|c_{j, k}^{i}\right|^{2}\right)=0$.

Then, for any integer $n$, and all $j \in\left\{j_{n}, \cdots, j_{n+1}-1\right\}$

$$
\mathbb{E}\left(\left|c_{j, k}^{i}\right|^{2}\right) \leq C_{0}^{2} \inf \left(2^{-j_{n} \bar{H}}, 2^{j_{n+1}(1-\bar{H})}, 2^{-j}\right)^{2}
$$

and by the same method as above it follows that for any $\gamma>1$

$$
\sup _{x \in \mathbb{R}^{d}} \sup _{|h| \leq 2^{-j}}|f(x+h)-f(x)| \leq C_{0} \inf \left(2^{-j_{n} \bar{H}}, 2^{j_{n+1}(1-\bar{H})}, 2^{-j}\right) j^{\gamma+\frac{1}{2}} .
$$

We then deduce almost surely the two upper bounds in equations (5.8), (5.9) and

$$
\bar{H} \geq \mathcal{I}_{\mathrm{loc}}\left(B_{\mathbf{H}, \sigma}^{1}\right)=\mathcal{I}_{\mathrm{loc}}\left(B_{\mathbf{H}, \sigma}\right) .
$$

We now want to bound the local uniform irregularity exponent of LFBM.

By the same proof as in the previous section we obtain that almost surely there exists a constant almost surely positive $C_{0}(\omega)$ and an integer $n_{0}(\omega)$ such that

$$
\forall n \geq n_{0}(\omega), \max _{|k| \leq \ell_{n} 2^{\ell_{n} d}}\left|c_{\ell_{n}, k}^{i}\right| \geq C_{0}(\omega) 2^{-\ell_{n} \underline{H}} .
$$


Hence:

- For any integer $n$ and any $j \in\left\{j_{n}, \ldots, \ell_{n}\right\}$,

$$
\max \left(\sup _{\ell \geq j}\left|c_{\ell, k}^{i}\right|, 2^{-j} \sup _{\ell \leq j} 2^{\ell}\left|c_{\ell, k}^{i}\right|\right) \geq C_{0}(\omega) 2^{-\ell_{n}} \underline{H} \geq C_{0}(\omega) 2^{-j \bar{H}} .
$$

- For any integer $n$ and any $j \in\left\{\ell_{n}+1, \ldots, j_{n+1}-1\right\}$

$$
\max \left(\sup _{\ell \geq j}\left|c_{\ell, k}^{i}\right|, 2^{-j} \sup _{\ell \leq j} 2^{\ell}\left|c_{\ell, k}^{i}\right|\right) \geq C_{0}(\omega) 2^{-j} 2^{\ell_{n}(1-\underline{H})} \geq C_{0}(\omega) 2^{-j \bar{H}} .
$$

Then using the wavelet criterium, we can conclude.

Moreover, using for any integer $j$,

$$
\left|c_{j, k}^{i}\right| \leq C_{\psi, \omega} \sup _{x \in \mathbb{R}^{d}} \sup _{|h| \leq 2^{-j}}\left|B_{\underline{H}, \tau}(x+h)-B_{\underline{H}, \tau}(x)\right|
$$

and (6.7) we deduce the lower bound in (5.9).

Since for any integer $n, \underline{H} \ell_{n}=\bar{H} j_{n}$ and

$$
\sup _{x \in \mathbb{R}^{d}} \sup _{|h| \leq 2^{-\ell_{n}}}\left|B_{\underline{H}, \tau}(x+h)-B_{\underline{H}, \tau}(x)\right| \leq \sup _{x \in \mathbb{R}^{d}} \sup _{|h| \leq 2^{-j n}} \mid B_{\underline{H}, \tau}(x+h)-B_{\underline{H}, \tau}(x)
$$

the lower bound in equation (5.8) follows.

Acknowledgements. The author would like to thank the anonymous referees whose valuable comments greatly improve the earlier version of this work.

\section{REFERENCES}

[1] A. Ayache and J. Lévy-Véhel, Generalized Multifractional Brownian Motion: definition and preliminary results, in Fractals Theory and applications in engineering, edited by M. Dekking, J. Lévy-Véhel, E. Lutton and C. Tricot. Springer (1999) 17-32.

[2] J.M. Bardet and P. Bertrand, Definition, properties and wavelets analysis of Multiscale Fractional Brownian Motion. Fractals 15 (2007) 73-87.

[3] J.M. Bardet, G. Lang, G. Oppenheim, A. Phillipe, S. Stoev and M.S. Taqqu, Generators of long-range dependent processes: A survey, in Theory and Applications of Long Range Dependance, edited by P. Doukhan M. Oppenheim and G. Taqqu. Birkäuser (2003) 579-623.

[4] M. Basseville and I. Nikiforov, Detection of abrupt changes-Theory and applications. Prentice-Hall (1993).

[5] A. Benassi and S. Deguy, Multi-scale Fractional Motion: definition and identification, Preprint LAIC (1999).

[6] A. Benassi, S. Jaffard and D. Roux, Elliptic Gaussian random processes. Revista Matematica Iberoamericana 13 (1997) 19-90.

[7] J. Beran, Statistics for Long-Memory processes. Chapman and Hall, London, UK (1994).

[8] Z. Ciesielski, G. Kerkyacharian and B. Roynette, Quelques espaces fonctionnels associés à des processus Gaussiens. Stud. Math . 107 (1993).

[9] M. Clausel, More about uniform irregularity: the wavelet point of view. Preprint (2008).

[10] J.J. Collins and C.J. De Luca, Open loop and closed loop control of posture: a random walk analysis of center of pressure trajectories, Exp. Brain Res. 9 (1993) 308-318.

[11] H. Csörgö and L. Horvath, Non parametric method for change point problems in Handbook of statistics, edited by P.R. Krishnaiah and C.R. Rao. Elsevier, New York 7 (1988) 403-425.

[12] R.B. Davies and D.S. Harte, Tests for Hurst effect. Biometrika 74 (1987) 95-101.

[13] C.R. Dietrich and G.N. Newsam, Fast and exact simulation of stationary Gaussian processes through circulant embedding of the covariance matrix. SIAM J. Sci. Comput. 18 (1997) 1088-1107.

[14] K. Falconer, Fractal Geometry. John Wiley and Sons (1990).

[15] K. Falconer, Tangent Fields and the local structure of random fields. J. Theor. Prob. 15 (2002) 731-750.

[16] K. Falconer, The local structure of random processes. J. London Math. Soc. 67 (2003) 657-672. 
[17] U. Frisch, Turbulence, the legacy of A.N. Kolmogorov. Cambridge University Press (1995).

[18] J.P. Kahane, Geza Freud and lacunary Fourier series. J. Approx. Theory 46 (1986) 51-57.

[19] I. Karatzas and S.E. Shreve, Brownian Motion and stochastic calculus. Springer-Verlag (1988).

[20] A.N. Kolmogorov, Wienersche Spiralen und einige andere interessante Kurven im Hilbertschen Raum. C. R. Acad. Sci. URSS 26 (1940) 115-118.

[21] J. Lévy-Vehel and R.F. Peltier, Multifractional Brownian Motion: definition and preliminary results, Rapport de recherche de l'INRIA n ${ }^{\circ} 2645$ (1995).

[22] S. Mallat, A wavelet tour of signal processing. Academic Press (1998).

[23] Y. Meyer, Ondelettes et opérateurs. Hermann (1990).

[24] Y. Meyer, F. Sellan and M.S. Taqqu, Wavelets, generalized white noise and fractional integration: the synthesis of Fractional Brownian Motion. J. Fourier Anal. Appl. 5 (1999) 465-494.

[25] B.M. Mandelbrot and J. Van Ness, Fractional Brownian Motion, fractional noises and applications. SIAM Rev. 10 (1968) 422-437.

[26] W. Willinger, M.S. Taqqu and V. Teverosky, Stock market price and long-range dependence. Finance and Stochastics 1 (1999) $1-14$.

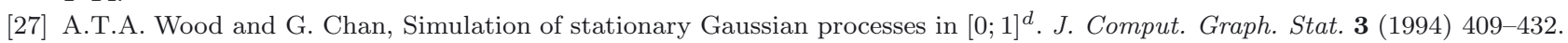

